\title{
Perceptions de mères d'enfant présentant un trouble du spectre de l'autisme ayant reçu une intervention comportementale intensive : leurs ressources et leurs défis Challenges and resources of mothers of children with autism spectrum disorder in the context of intensive behavioural intervention
}

\author{
Nadia Abouzeid et Nathalie Poirier
}

Volume 43, numéro 2, 2014

URI : https://id.erudit.org/iderudit/1061182ar

DOI : https://doi.org/10.7202/1061182ar

Aller au sommaire du numéro

Éditeur(s)

Revue de Psychoéducation

ISSN

1713-1782 (imprimé)

2371-6053 (numérique)

Découvrir la revue

Citer cet article

Abouzeid, N. \& Poirier, N. (2014). Perceptions de mères d'enfant présentant un trouble du spectre de l'autisme ayant reçu une intervention comportementale intensive : leurs ressources et leurs défis. Revue de psychoéducation, 43(2), 201-233. https://doi.org/10.7202/1061182ar

\section{Résumé de l'article}

L'objectif général de l'étude est de décrire l'expérience de mères d'enfant présentant un trouble du spectre de l'autisme (TSA) ayant reçu une intervention comportementale intensive (ICI). Plus spécifiquement, leurs défis, leurs ressources (leur savoir, leur sentiment d'auto-efficacité, leur implication et leurs stratégies d'adaptation) et le soutien reçu ont été explorés. Pour ce faire, 15 participantes ont rempli une fiche signalétique et ont été interrogées dans le cadre d'une entrevue semi-structurée. Les données recueillies indiquent que ces mères font face à de nombreux défis découlant du diagnostic de leur enfant. Pour pallier ces difficultés, elles utilisent principalement leurs ressources personnelles et elles déploient de nouvelles stratégies d'adaptation. De plus, leur savoir parental reflète une compréhension du TSA et de l'ICI qui est variable et qui semble associée à la formation et au soutien reçu ainsi qu'à leur implication au sein de l'intervention.
Tous droits réservés ( $\odot$ La Corporation de la Revue Canadienne de Psycho-Éducation, 2014
Ce document est protégé par la loi sur le droit d'auteur. L'utilisation des services d'Érudit (y compris la reproduction) est assujettie à sa politique d'utilisation que vous pouvez consulter en ligne. 


\section{Perceptions de mères d'enfant présentant un trouble du spectre de l'autisme ayant reçu une intervention comportementale intensive : leurs ressources et leurs défis.}

\section{Challenges and resources of mothers of children with autism spectrum disorder in the context of intensive behavioural intervention.}

\section{N. Abouzeid ${ }^{1}$ \\ N. Poirier ${ }^{1}$}

Département de psychologie, Université du Québec à Montréal (UQAM)

\section{Correspondance :}

Nadia Abouzeid nadia.abouzeid@gmail.com

\section{Résumé}

L'objectif général de l'étude est de décrire l'expérience de mères d'enfant présentant un trouble du spectre de l'autisme (TSA) ayant reçu une intervention comportementale intensive (ICI). Plus spécifiquement, leurs défis, leurs ressources (leur savoir, leur sentiment d'auto-efficacité, leur implication et leurs stratégies d'adaptation) et le soutien reçu ont été explorés. Pour ce faire, 15 participantes ont rempli une fiche signalétique et ont été interrogées dans le cadre d'une entrevue semi-structurée. Les données recueillies indiquent que ces mères font face à de nombreux défis découlant $d u$ diagnostic de leur enfant. Pour pallier ces difficultés, elles utilisent principalement leurs ressources personnelles et elles déploient de nouvelles stratégies d'adaptation. De plus, leur savoir parental reflète une compréhension du TSA et de I'ICI qui est variable et qui semble associée à la formation et au soutien reçu ainsi qu'à leur implication au sein de l'intervention.

Mots-clés : trouble du spectre de l'autisme, interventioncomportementaleintensive,stratégies d'adaptation, soutien, expérience maternelle.

\section{Abstract}

The general objective of this study is to describe the experience of mothers of children with autism spectrum disorder (ASD) in the context of intensive behavioural intervention (IBI). More specifically, their challenges, resources (knowledge, self-efficacy, involvement and adaptive strategies) and support were examined. In order to achieve this, descriptive forms were completed and semi structured interviews were held with 15 participants. Results of this study show that these mothers face many challenges related to the diagnosis of their child. They learn to overcome these challenges by using their individual resources and by exerting new coping strategies. Their 


\section{2}

understanding of $I B I$ and $A S D$ is variable and seems to be related to the training and support provided as well as their involvement during the intervention.

Keywords: Autism spectrum disorder, intensive behavioural intervention, coping strategies, support and mother's experience.

\section{Contexte théorique et objectifs}

Le trouble du spectre de l'autisme ${ }^{1}$ (TSA), caractérisé par sa nature neurodéveloppementale, désigne une altération persistante du développement, notamment sur le plan de la communication sociale et sur celui des comportements et des intérêts. Le TSA comprend trois niveaux de sévérité qui se distinguent en fonction du degré de soutien requis. L'élaboration des caractéristiques relatives aux deux sphères déficitaires permet de déterminer le niveau de sévérité du TSA. Ainsi, le premier niveau requiert un besoin de soutien, le niveau deux, un besoin substantiel de soutien et le niveau trois, un besoin très substantiel de soutien (American Psychiatric Association, 2013). D’après les études épistémologiques les plus récentes, près de $1 \%$ de la population serait atteinte d'un TSA (American Psychiatric Association, 2013; Fombonne, Zakarian, Bennett, Meng, \& McLeanHeywood, 2006; Lamontagne, 2011; Lazoff, Lihong, Piperni, \& Fombonne, 2010; Newschaffer et al., 2007; Saracino, Noseworthy, Steiman, Reisinger, \& Fombonne, 2010; Weintraub, 2011). Par ailleurs, le Center for Disease Control and Prevention (CDC) (2012) estime le taux de prévalence à 1/88. Le TSA induit des changements familiaux pouvant altérer de manière importante la qualité de vie de la personne atteinte du trouble, de sa fratrie et de ses parents. Les parents devant s'ajuster à cette réalité déploient de nombreux efforts pour surmonter les obstacles découlant du diagnostic. Ils doivent également faire des choix quant au type d'intervention à privilégier pour contribuer à l'éducation de leur enfant et à l'amélioration de sa qualité de vie (Allik, Larsson, \& Smedje, 2006; Eisenhower, Baker, \& Blacher, 2005; Giarelli, Souders, Pinto-Martin, Bloch, \& Levy, 2005; Sénéchal \& des RivièresPigeon, 2009; Twoy, Connolly, \& Novak, 2007; Wintgens \& Hayez, 2006). Peu de données existent sur le vécu des parents d'enfant présentant un TSA ayant reçu une intervention comportementale intensive $(\mathrm{ICI})$ en contexte québécois. II importe de s'y intéresser afin de mieux comprendre la manière dont les familles perçoivent leur situation et la façon dont l'ICI peut contribuer à leur bien-être. Puisque les parents sont les principaux acteurs impliqués dans l'éducation de leur enfant, rendre compte de leur expérience permettra d'améliorer la compréhension de leur vécu et d'orienter le développement et la bonification des services offerts.

L'objectif de cette étude est de décrire l'expérience des mères d'enfant présentant un TSA ayant reçu une $\mathrm{ICI}$ en identifiant les défis qu'elles ont rencontrés depuis le diagnostic, leurs ressources personnelles ainsi que les diverses formes de soutien que l'ICl leur a procurées et qui les ont aidées à composer avec leurs

1. Ce terme et son acronyme TSA seront employés dans ce texte pour alléger la lecture et pour favoriser la compréhension des lecteurs. Leur utilisation fera parfois référence à l'appellation trouble envahissant du développement qui est largement utilisé dans les documents ministériels, et ce même s'il existe des distinctions dans la définition de ces termes. 
responsabilités parentales et les diverses difficultés auxquelles elles font face. Le présent article décrira d'abord I'ICI et ensuite, l'expérience des parents d'enfant présentant un TSA, plus spécifiquement la détresse psychologique, l'implication parentale, le sentiment d'auto-efficacité, le savoir parental, le soutien et les stratégies d'adaptation. Ensuite, la méthode, les résultats, la discussion et la conclusion seront présentés.

\section{Intervention comportementale intensive ${ }^{2}$}

L'ICI renvoie aux programmes d'intervention pour les enfants présentant un TSA. L'ICI s'inspire des travaux de Lovaas (1987) qui a élaboré un programme d'intervention pour les enfants présentant un TSA en se référant aux principes fondamentaux de l'analyse appliquée du comportement (AAC) (angl. Applied Behaviour Analysis, ABA). L'AAC est une discipline appliquée, fondée sur les postulats du conditionnement opérant (Forget, Schuessler, Paquet, \& Giroux, 2005; McGahan, 2001).

À ce jour, l'ICl demeure l'intervention ayant le plus d'appuis empiriques démontrant son efficacité auprès d'enfants qui sont atteints d'un TSA (Cohen, Amerine-Dickens, \& Smith, 2006; Eikeseth, 2011; Eldevik et al., 2009, 2010; Larsson, 2008, 2010; Lovaas, 1987; Lovaas et al., 1973; Maglione, Gans, Das, Timbie, \& Kasari, 2012; Makrygianni \& Reed, 2010; Peters-Scheffer, Didden, Korzilius, \& Sturmey, 2011; Reichow, 2012; Virués-Ortega, 2010; Warren et al., 2011). Plusieurs études, méta-analyses, revues systématiques et revues de métaanalyses démontrent que I'ICI permet l'amélioration du langage (Maglione et al., 2012; Warren et al., 2011), du quotient intellectuel (Eldevik et al., 2009; Maglione et al., 2012; Reichow, 2012; Warren et al., 2011) et des comportements adaptatifs (Eldevik et al., 2009; Maglione et al., 2012; Reichow, 2012; Virués-Ortega, 2010; Warren et al., 2011). Bien qu'elles soient moins nombreuses, certaines études ont également évalué l'efficacité de l'ICl en contexte communautaire (Blacklock, Weiss, Perry, \& Freeman, 2012; Eikeseth, Klintwall, Jahr, \& Karlsson, 2012; Eldevik, Hastings, Jahr, \& Hughes, 2012; Fernell et al., 2011). Celles-ci font état de progrès similaires sur le plan des comportements adaptatifs (Eikeseth et al., 2012; Eldevik et al., 2012; Fernell et al., 2011; Perry et al., 2008), des symptômes autistiques (Perry et al., 2008) et du fonctionnement intellectuel (Eldevik et al., 2012; Perry et al., 2008). Cependant, plusieurs difficultés ont été soulevées quant à l'implantation et peuvent s'expliquer, entre autres variables, par un roulement élevé du personnel (Gamache, Joly, \& Dionne, 2011) et une faible participation des parents à l'intervention (Eikeseth et al., 2012; Gamache et al., 2011).

2. Plusieurs appellations et acronymes sont utilisés dans la littérature, à tort ou à raison, pour désigner l'intervention comportementale intensive (ICl), soit l'intervention comportementale précoce (ICP), l'intervention intensive comportementale (IIC), l'intervention comportementale intensive précoce (ICIP), l'Early Intensive Behavioral Intervention (EIBI), I'Intensive Behavioural Intervention (IBI), l'approche d'analyse appliquée du comportement (AAC) et l'Applied Behaviour Analysis $(A B A)$. Pour alléger le présent document et favoriser la compréhension des lecteurs, les termes Intervention comportementale intensive et l'acronyme $\mathrm{ICl}$ seront privilégiés, mais surtout puisqu'ils reflètent davantage la réalité québécoise. 
La perception des parents en ce qui concerne les programmes d'intervention a fait l'objet de quelques études (Blacklock et al., 2012; Bowker, D'Angelo, Hicks, \& Wells, 2011). L'évaluation de l'ICI par les parents semble essentielle, puisqu'elle peut influencer leur choix d'intervention, leur degré d'implication et, plus encore, la qualité de l'intervention (Blacklock et al., 2012; Solish \& Perry, 2008). Les résultats d'études canadiennes révèlent une perspective positive des parents quant aux progrès perçus chez leur enfant suite à l'ICl (Blacklock et al., 2012; Bowker et al., 2011; Courcy \& des Rivières-Pigeon, 2012). Parallèlement, des chercheurs ont démontré que l'AAC constitue l'approche privilégiée par les parents d'enfant présentant un TSA (Bowker et al., 2011). Selon cette étude, le choix de l'intervention des parents ne repose pas nécessairement sur les données probantes. Ce serait plutôt les informations obtenues quant aux interventions, l'accessibilité de l'intervention et les attentes en regard des effets de l'intervention qui orientent les parents dans leur décision (Tzanakaki et al., 2012).

Corrélativement à ce qui précède, certains auteurs rappellent l'importance de s'intéresser aux conséquences d'une $\mathrm{ICl}$ sur les parents et la fratrie afin de comprendre pleinement l'efficacité de l'intervention (Grindle \& Remington, 2014; Karst \& Van Hecke, 2012). II est important de vérifier la perception des parents quant aux effets de l'ICI sur l'enfant, mais également sur la famille. II est possible que l'intervention s'additionne aux défis que rencontrent ces parents à la suite du diagnostic de leur enfant. II s'avère essentiel de reconnaiître les besoins spécifiques des parents et d'y porter une attention particulière, sinon il se peut que l'intervention ne produise pas les effets escomptés (Karst \& Van Hecke, 2012; Maglione et al., 2012). Dans certains cas, elle pourrait même nuire au développement de l'enfant et à la qualité de vie des membres de sa famille (Grindle \& Remington, 2014; Karst \& Van Hecke, 2012). L'intervention auprès des personnes ayant un TSA requiert l'implication des parents de diverses façons (Granger, des Rivières-Pigeon, Sabourin, \& Forget, 2010). La participation parentale peut contribuer à des gains significatifs chez l'enfant, mais également produire des effets positifs sur plusieurs variables parentales. Parmi celles-ci se trouvent notamment une meilleure connaissance du TSA (McConachie \& Diggle, 2007), la hausse du sentiment d'autoefficacité, la baisse du niveau de stress et l'amélioration de la relation conjugale (Karst \& Van Hecke, 2012).

\section{Expérience des parents d'enfant présentant un TSA}

Détresse psychologique. Le TSA et les divers troubles qui y sont associés altèrent le fonctionnement de la personne qui en est atteinte et celui de la famille entière (American Psychiatric Association, 2013; Bandini et al., 2010; Cotton \& Richdale, 2006; Giarelli et al., 2005; Gurney, McPheeters, \& Davis, 2006; Lord \& McGee, 2001; Martins, Young, \& Robson, 2008; Matson \& Nebel-Schwalm, 2007). Pour pallier les difficultés découlant du diagnostic, de nombreux changements sont requis au quotidien, ce qui ajoute au stress vécu par les parents (Courcy $\&$ des Rivières-Pigeon, 2013; Estes et al., 2009; Poirier \& Goupil, 2008). À cet égard, plusieurs études révèlent un niveau de détresse psychologique important chez les parents d'enfant présentant un TSA (Allik et al., 2006; Courcy \& des RivièresPigeon, 2013; Eisenhower et al., 2005; Montes \& Halterman, 2007; Rivard, Terroux, Parent-Boursier, \& Mercier, 2014; Sénéchal \& des Rivières-Pigeon, 2009), une 
qualité de vie moindre ainsi qu'une utilisation plus faible des ressources et des stratégies d'adaptation (Allik et al., 2006). L'état de santé mentale et émotionnelle des mères s'avère également inférieur (Montes \& Halterman, 2007). Des taux de dépression (Courcy \& des Rivières-Pigeon, 2013) et de stress (Eisenhower et al., 2005; Hayes \& Watson, 2013; Rivard et al., 2014) plus élevés ont été observés chez les mères d'enfant présentant un TSA en comparaison avec les mères d'enfant ayant des difficultés de développement appartenant à une autre catégorie. L'étude de Rivard et ses collaborateurs (2014) est une des rares à avoir examiné le taux de stress chez les pères, qui atteint un seuil encore plus élevé que celui des mères de leur échantillon.

Plusieurs chercheurs se sont intéressés aux variables déterminantes du stress parental (Courcy \& des Rivières-Pigeon, 2013; Davis \& Carter, 2008; Estes et al., 2009; Hastings \& Johnson, 2001; Mekki, 2012; Sénéchal \& des Rivières-Pigeon, 2009). Les problèmes financiers, les difficultés conjugales, la monoparentalité, les difficultés de conciliation travail-famille ont été relevés (Courcy \& des Rivières-Pigeon, 2013). D'autres auteurs ont identifié des facteurs plus spécifiques aux responsabilités et aux rôles parentaux, à la relation parentenfant et aux caractéristiques de l'enfant ayant un TSA (Davis \& Carter, 2008; Mekki, 2012; Montes \& Halterman, 2007; Rivard et al., 2014). Plus précisément, la sévérité de la symptomatologie de l'enfant, son âge, son sexe, les déficits sur le plan du fonctionnement intellectuel, langagier et social ainsi que les comportements problématiques sont associés au stress parental (Davis \& Carter, 2008; Estes et al., 2009; Hastings \& Johnson, 2001; Mekki, 2012; Rivard et al., 2014). Le délai d'attente pour les services d'intervention ainsi que les comportements adaptatifs peuvent également avoir une incidence sur le taux de stress des parents. II semble qu'il y ait certaines divergences dans le développement du stress chez le père et chez la mère. Le stress de la mère serait associé à l'âge de l'enfant alors que pour le père, il serait non seulement lié à l'âge, mais également au sexe de l'enfant (Rivard et al., 2014).

Implication parentale. Les études examinant les effets des interventions et leur efficacité mettent en lumière l'importance d'inclure la famille dans les interventions puisque cette dernière est reconnue comme étant au centre du développement de l'enfant (Chrétien, Connolly, \& Moxness, 2003; des RivièresPigeon \& Courcy, 2014; Guralnick, 2000, 2001, 2005; Minjarez, Williams, Mercier, \& Hardan, 2011; National Research Council Committee on Educational Interventions for Children with Autism, 2001). Toutefois, il semble que la réalité actuelle ne reflète pas ce qui est conceptuellement et théoriquement recommandé. D'ailleurs, le rapport d'enquête du protecteur du citoyen (2009) révèle que la présence du parent n'est pas toujours sollicitée. De plus, certains auteurs notent l'absence d'un modèle systémique des services d'intervention, ce qui peut nuire au développement optimal des enfants vulnérables, en plus d'ajouter aux défis que rencontre leur famille (Chrétien et al., 2003; Guralnick, 2000, 2001, 2005). Aux États-Unis, les lois sur l'éducation d'une personne ayant un handicap ou un trouble (Education of the Handicapped Act Amendments of 1986 (PL-99-457) et Individuals with Disabilities Act Amendments of 1997 PL 105-17, Section 631) encouragent l'intervention précoce des enfants ayant une incapacité et incitent la participation des familles dans leur milieu naturel dans le but de favoriser à la fois, la diminution des 


\section{6}

symptômes attribués au TSA, l'actualisation du potentiel de développement ainsi que l'intégration de l'enfant (California Departments of Education, 1997; Guralnick, 2000; McGee, Morrier, \& Daly, 1999; New York State Department of Health, 1999). De plus, la collaboration de la famille dans l'intervention est une pratique courante aux États-Unis puisque les professionnels du milieu sont tenus par la loi intitulée Individualized Family Service Plan Public Law 99-457 de participer à la conception du plan de services individualisé à la famille (PSIF) (California Departments of Education, 1997). Au Québec, la Loi sur les services de santé et les services sociaux (LSSSS) exige également l'élaboration d'un plan de services individualisé (PSI) permettant de répartir certains des rôles et des responsabilités à la personne et à ses proches (Gouvernement du Québec, 2013). Toutefois l'offre de services varie selon les régions (Protecteur du citoyen, 2012). En contexte d'ICl, plusieurs études ont démontré que l'implication parentale facilite la généralisation et le maintien des acquis (Lovaas et al., 1973; Matson, Mahan, \& Matson, 2009; Solish \& Perry, 2008). Par ailleurs, le programme de Lovaas (1987) requiert la participation du parent en tant que cothérapeute auprès de son propre enfant, et ce à raison de 10 heures par semaine. Pour ce faire, les parents bénéficient d'une formation intensive et continue qui leur enseigne les principes fondamentaux de l'AAC et des moyens d'intervention à appliquer. Pour favoriser leur implication, le programme exige que le parent soit présent à au moins une séance d'ICI par semaine (Hayward, Gale, \& Eikeseth, 2009). La formation des parents produit des effets positifs sur leurs habiletés parentales, leur savoir parental et la diminution de leur stress (Minjarez et al., 2011; Solish \& Perry, 2008). Par conséquent, des effets positifs se perçoivent chez l'enfant, notamment des améliorations sur le plan du langage ainsi qu'une diminution des comportements problématiques (Crockett, Fleming, Doepke, \& Stevens, 2007; McConachie \& Diggle, 2007). Parallèlement, Solish et Perry (2008) soulèvent l'existence d'un lien entre la participation des parents, leur compréhension de l'ICl et du TSA et leur sentiment d'auto-efficacité. Selon ces auteurs, l'implication parentale désigne à la fois la collaboration des parents avec l'équipe d'intervention et l'intervention auprès de leur enfant.

L'étude exploratoire de Granger et ses collaborateurs (2010), effectuée auprès de 13 mères d'enfant présentant un TSA ayant participé à un programme d'ICl en centre de réadaptation en déficience intellectuelle et en troubles envahissants du développement (CRDITED) montre que la forme d'implication la plus adoptée est l'application, au quotidien, de certains principes d'intervention, mais que les mères effectuent également de la généralisation des acquis et des séances structurées d'intervention comme le recommande la programmation d'ICI (Lovaas, 1987; Lovaas et al., 1973). De plus, les propos recueillis soulignent également le rôle important des intervenants et de la qualité de la relation établie avec la famille dans la façon dont les parents choisissent de s'impliquer dans l'ICI (Granger et al., 2010).

Dans le même ordre d'idées, peu d'études recensent les formations offertes aux parents du Québec pour acquérir une meilleure connaissance de I'ICl et du rôle qu'ils doivent jouer dans l'intervention. Actuellement, certains établissements universitaires offrent des programmes de formation sur l'intervention comportementale auprès des personnes ayant un TSA, mais certains ne s'adressent qu'aux intervenants des CRDITED et ne sont donc pas accessibles aux parents. 
Par exemple, afin d'accéder aux programmes de formation offerts par l'Université du Québec à Montréal, un parent doit avoir complété un baccalauréat (Direction générale de la formation continue \& Université Laval, 2003a, 2003b; FQCRDITED, 2003). Outre la formation universitaire, quelques Centre de santé et de services sociaux (CSSS) et CRDITED, des associations de parents ainsi que les cliniques de psychologie spécialisées en TSA offrent des formations de courte durée sur des thématiques particulières. Par exemple, certaines enseignent les techniques de base de l'ICl.

Sentiment d'auto-efficacité parentale. Le sentiment d'auto-efficacité, défini comme étant la perception d'une personne quant à ses habiletés dans divers domaines, résulte des réussites et des échecs vécus par cette dernière et tend à orienter ses comportements ultérieurs (Bandura, 1977, 1989). Le sentiment d'autoefficacité parentale désigne la perception que développe une personne dans son rôle de parent (Jones \& Prinz, 2005). Ce concept a notamment été étudié chez des populations de parents d'enfant ayant des troubles d'apprentissage. Ces études concluent qu'un sentiment d'auto-efficacité élevé contribue à l'amélioration de certaines formes d'implication dans l'intervention de leur enfant, telles que le bénévolat et la participation à des activités éducatives autres que les devoirs et les leçons (Anderson \& Minke, 2007; Green, Walker, Hoover-Dempsey, \& Sandler, 2007). Les études effectuées auprès de parents d'enfant présentant un TSA confirment les résultats de ces recherches (Hastings \& Symes, 2002; Kuhn \& Carter, 2006; Solish \& Perry, 2008). Par exemple, Solish et Perry (2008) montrent que le sentiment d'auto-efficacité est la variable la plus fortement liée à l'implication des parents en contexte d'ICI. Parallèlement, la perception positive de l'intervention est associée à un sentiment d'auto-efficacité parental plus élevé. Des liens entre le sentiment d'auto-efficacité et le stress vécu par les parents d'enfant ayant un TSA ont également été établis (Hastings \& Symes, 2002; Kuhn \& Carter, 2006). Bien que le sentiment d'auto-efficacité soit une variable déterminante, il demeure peu documenté dans le contexte de l'ICl.

Savoir parental. Tel que le précisent Solish et Perry (2008) ainsi que McConachie et Diggle (2001), l'information, la compréhension et les croyances que détiennent les parents concernant le TSA et l'intervention sont des variables clés dans leur façon de composer avec l'annonce du diagnostic de TSA et d'intervenir auprès de leur enfant. Certaines études se sont penchées sur les connaissances acquises par les parents à l'annonce du diagnostic. L'étude de Poirier et Goupil (2008) réalisée auprès de 88 familles québécoises recrutées par le biais des CRDITED et de sites internet met de l'avant la bonne satisfaction des parents quant à l'information reçue en regard du diagnostic, du pronostic et des services offerts. $\grave{A}$ l'inverse, certains auteurs soulignent plutôt l'insatisfaction des parents qui déplorent la quantité de procédures administratives et se plaignent de la qualité de la relation avec les multiples intervenants (Chrétien et al., 2003). L'étude de Veyssière et de ses collaborateurs (2010), réalisée en France, indique des lacunes sur le plan de la compréhension du TSA à la suite de l'annonce du diagnostic. D'ailleurs, la majorité des parents ignorent, méconnaissent le TSA ou détiennent de l'information très négative par rapport au trouble à ce moment donné. L'une des stratégies adoptées par les parents de cette étude, pour contrer cette méconnaissance, est la recherche d'informations qui leur permettrait de s'adapter à l'annonce d'un tel diagnostic 


\section{8}

(Veyssière, Bernadoy, Dagot, \& Goussé, 2010). De plus, il a été démontré que le besoin d'être informé quant aux caractéristiques et aux besoins spécifiques de leur enfant, la détresse psychologique qu'amène le diagnostic, le manque d'accessibilité aux ressources pour répondre aux besoins de l'enfant ainsi que la perception négative de leurs compétences parentales constituent des stresseurs pouvant influencer la qualité de vie des familles ayant un enfant présentant un TSA (Sabourin, des Rivières-Pigeon, \& Granger, 2011). À ce jour, les études sont peu nombreuses à examiner le besoin d'informations des parents et le type de savoir que ces parents cherchent à acquérir, d'autant plus que la majorité d'entre elles ne traitent pas en profondeur des connaissances que possèdent les parents quant au TSA et aux services offerts (Beaud \& Quentel, 2011a, 2011b). L'étude de Poirier et Goupil (2008) s'est intéressée aux connaissances des parents au sujet du TSA. Elle indique que les deux tiers des parents détenaient de l'information avant la naissance de leur enfant. Ils l'auraient acquise grâce à leur relation avec des personnes ayant un diagnostic, par les médias, dans le cadre de leur travail ou de leurs études ou par des membres de leur réseau social. Aucune étude n'a encore analysé en profondeur le type d'information que les parents peuvent acquérir en contexte d'ICI concernant le diagnostic de TSA et l'intervention elle-même.

Soutien et ressources. La littérature décrit l'ampleur des difficultés vécues par les familles d'enfants présentant un TSA, mais les besoins spécifiques de ces dernières, les stratégies d'adaptation qu'elles utilisent, les ressources disponibles ainsi que le soutien auquel elles ont accès depuis l'annonce du diagnostic et en contexte d'ICl demeurent toutefois peu documentés, d'autant plus que la majorité des études traitant de ces thèmes sont américaines. II semble que les différentes formes de soutien offertes aux familles constituent des ressources contribuant à réduire le niveau de stress lié aux difficultés vécues par ces familles et sont, par le fait même, associées à leur bien-être (Benson \& Dewey, 2008; Cappe, Wolff, Bobet, \& Adrien, 2011; Montes \& Halterman, 2007; Siklos \& Kerns, 2006; Twoy et al., 2007; White \& Hastings, 2004). D'abord, le type de soutien peut être informel ou formel.Le soutien informel renvoie davantage au soutien offert par le réseau social (p.ex., conjoint, famille élargie, amis, groupe religieux), alors que le soutien formel réfère à celui prodigué par les différents services et professionnels. Ce type de soutien joue un rôle important dans l'accès à l'information pour les parents, dans la résolution de problèmes, dans le développement de relations entre les familles ainsi que dans l'établissement du partenariat entre les services et les familles. Bien que les études démontrent clairement un lien entre le soutien informel et la réduction du niveau de stress chez ces familles, les effets du soutien formel sur le stress demeurent moins évidents. Le soutien formel serait davantage lié aux besoins des enfants (White \& Hastings, 2004). Ces soutiens peuvent aussi être catégorisés selon leur fonction dans la vie de la famille, qu'ils soient de nature instrumentale (p.ex., soutien financier, soutien lié aux tâches ménagères), émotive (p. ex., avoir quelqu'un à qui se confier), informative (p.ex., recevoir de l'information sur le TSA) ou récréative (p.ex., sorties avec des amis) (Benson \& Dewey, 2008; Bromley, Hare, Davison, \& Emerson, 2004; Cappe et al., 2011; des Rivières-Pigeon \& Courcy, 2014; Siklos \& Kerns, 2006). Très peu d'études ont abordé le soutien et les ressources dont bénéficient les familles dans un contexte d'ICI. Au-delà de l'influence positive sur le développement du jeune, on peut supposer que l'ICl offre un type de soutien pouvant aider les parents à surmonter les difficultés qu'amène 
le diagnostic de TSA et à mieux intervenir auprès de leur enfant. Johnson et Hastings (2001) ont relevé des caractéristiques de l'ICl ayant un effet positif sur le bien-être des parents. Le soutien et l'implication de la part de l'équipe d'intervention ressortent comme les facteurs les plus utiles pour le développement de l'enfant. Le soutien apporté par d'autres familles recevant de l'ICl est également important (Beaud \& Quentel, 2011a).

\section{Méthode}

\section{Participantes}

Les 15 participantes recrutées sont des mères de 15 enfants présentant un TSA ayant reçu au moins huit mois consécutifs d'ICI en CRDITED. Quatre des enfants ont reçu des services complémentaires en clinique privée. L'âge des enfants varie de cinq à huit ans. Ils ont tous un diagnostic correspondant au TSA ${ }^{3}$ (TED-NS : $n=4$; autisme $: n=5$; TED $: n=2 ;$ TSA $: n=2$; TED/TSA $: n=2$ ). À la suite de l'ICl, ils sont scolarisés, soit en classe ordinaire $(n=4)$, en classe spécialisée $(n=7)$ ou en école spécialisée $(n=4)$. Ils étaient de niveau préscolaire ou de premier cycle du primaire au moment des entrevues. Pour la réalisation de ce projet de recherche, ce sont les mères plutôt que les pères qui ont été choisies, car elles répondent plus favorablement lorsqu'elles sont sollicitées pour des études et s'impliquent davantage dans les interventions (Benson, Karlof, \& Siperstein, 2008; Johnson \& Hastings, 2002). L'échantillon représente un groupe homogène puisque ces mères sont québécoises, qu'elles parlent français, qu'elles demeurent pour la plupart $(n=12)$ dans la grande région métropolitaine de Montréal et qu'elles vivent avec le père de l'enfant $(n=10)$. Quatre mères occupent un emploi à temps partiel, cinq travaillent à temps plein et six sont au foyer.

\section{Instruments}

Les instruments ayant servi à la collecte de données sont une fiche signalétique et un protocole d'entretien semi-structuré.

Fiche signalétique. Le formulaire utilisé compte 44 questions concernant les caractéristiques sociobiographiques des participantes. Des informations sur l'âge, le revenu, l'emploi, le niveau d'étude de la mère et de son conjoint ont été relevées. Les mères devaient également fournir des données sur leur situation familiale, le nombre d'enfants qu'elles ont, le diagnostic de l'enfant ainsi que l'ensemble des services obtenus par ce dernier.

Protocole d'entretien semi-structuré. Dans le cadre de cette étude, les entrevues semi-structurées visaient à recueillir des informations sur l'expérience des mères d'enfant présentant un TSA ayant reçu une ICI. Plus spécifiquement, les défis auxquels elles font face, leurs ressources personnelles ainsi que les stratégies qu'elles adoptent pour s'adapter à leur situation ont été explorés. Pour ce faire, une

3. Les diagnostics présentés font référence à ceux émis suite à l'évaluation diagnostique par le pédopsychiatre ou le psychologue. 


\section{0}

section du protocole d'entretien (expérience des mères) conçue dans le cadre d'un projet de recherche plus vaste a été utilisée pour répondre à l'objectif de la présente étude. Le protocole original comportant 65 questions a été élaboré en fonction des écrits sur le sujet et des objectifs ciblés. II s'articule autour 1) des caractéristiques de l'intervention, 2) de l'expérience des mères et 3) de la perception des effets de I'ICI. II a été soumis à une évaluation par quatre experts, soit deux psychologues exerçant en clinique privée et ayant une expertise dans le domaine du TSA et de I'ICl, une personne ressource au service régional de soutien et d'expertise à l'intention des élèves présentant un TSA des commissions scolaires francophones de Montréal ainsi qu'une spécialiste en activités cliniques travaillant en CRDITED auprès de cette clientèle. À la suite de l'évaluation des questions par les experts, la grille d'entrevue a été bonifiée et utilisée pour une préexpérimentation auprès de deux participantes. Des ajustements ont par la suite été effectués pour la version définitive du protocole.

Ainsi, des questions ouvertes relatives aux difficultés rencontrées, à leurs ressources personnelles (savoir parental, sentiment d'auto-efficacité, implication, stratégies d'adaptation) ainsi qu'au soutien reçu ont été posées aux participantes. Certaines sous-questions ont servi à préciser leur discours pendant l'entrevue. Le tableau 1 illustre certains exemples.

Tableau 1. Questions du protocole en lien avec les objectifs de l'étude

\section{Ressources personnelles}

Étiez-vous en quête d'information ? Que vouliez-vous savoir?

Quelles ont été vos principales sources d'information?

Selon vous, qu'est-ce qui aurait pu faciliter votre implication ou qu'est-ce qui aurait pu vous inciter à vous impliquer davantage?

À quel point vous sentiez-vous confiante d'appliquer les tâches relatives au programme d'ICI de votre enfant?

Comment vos ressources personnelles vous ont-elles aidé à composer avec les activités et les tâches liées à votre rôle de parent et au développement de votre enfant?

\footnotetext{
Soutien

Sur qui pouviez-vous compter réellement pour vous rassurer et vous encourager lorsque vous doutiez de vos capacités ?

Qui pouvait vous aider matériellement et/ou financièrement lors d'une période difficile?

Quelle formation avez-vous reçue en $\mathrm{ICI}$ ?

Quel était le type de soutien que vous offraient les intervenants?

Comment ce soutien vous a-t-il aidé à composer avec les activités et les tâches liées à votre rôle de parent et au développement de votre enfant ?
} 


\section{Procédures}

Le recrutement a été réalisé grâce à la collaboration des associations de parents, des réseaux sociaux et des personnes ressources des commissions scolaires de Montréal et de ses environs qui ont fait parvenir une lettre de sollicitation aux mères d'enfant ayant un TSA. Celle-ci décrivait les aspects importants de l'étude et invitait les mères intéressées à y participer à communiquer avec l'expérimentatrice. Des rencontres, d'une durée de 90 à 120 minutes, ont été réalisées au domicile des participantes ou dans un lieu qui leur convenait. Les entrevues, dirigées par l'expérimentatrice, ont été enregistrées sous forme audionumérique en vue de la transcription intégrale pour les fins de l'analyse qualitative. Les verbatim ont été rédigés par l'expérimentatrice et une assistance de recherche formée.

\section{Analyse de données}

Tel que mentionné précédemment, ce sont des entrevues semi-dirigées qui ont servi à recueillir les données traitées ensuite selon une analyse qualitative. Cela a permis de relever un contenu riche et détaillé difficilement accessible par l'utilisation de questionnaires ou d'échelles quantitatives. Les verbatim ont fait l'objet de plusieurs lectures et d'une analyse thématique (Miles \& Huberman, 2003; Paillé \& Mucchielli, 2008) pour laquelle le logiciel NVivo 9 a été utilisé. Les thèmes émergents ont été organisés en catégories pour représenter des idées distinctes. Par exemple, les mots suivants : « je me mobilise plus, je suis plus une militante " représentant les " caractéristiques et forces personnelles " ont permis de développer la catégorie "ressources personnelles ». La catégorisation a été révisée par une doctorante en psychologie afin d'obtenir un accord interjuges. La moyenne des coefficients de Kappa de Cohen en résultant est de 0,97 (ÉT=0,06).

\section{Résultats}

Les données recueillies ont été divisées selon les trois objectifs ciblés. D'abord, les défis rencontrés depuis le diagnostic seront présentés. Ensuite, les ressources personnelles des mères seront exposées. Finalement, le soutien reçu par les mères ainsi que leurs relations avec les éducatrices ${ }^{4}$ seront décrits.

\section{Défis rencontrés par les mères}

Acceptation du diagnostic. L'obtention du diagnostic est vécue de diverses façons chez les mères interrogées. Certaines éprouvent toujours de la difficulté à composer avec le diagnostic de leur enfant $(n=5)$. Elles ont été choquées $(n=4)$ par cette nouvelle qui a suscité de nombreuses inquiétudes par rapport à l'avenir de leur enfant $(n=8)$. À ce sujet, une des participantes s'exprime :

4. Le féminin est employé pour désigner les personnes intervenant (éducatrices, travailleuses sociales) auprès des enfants de cette étude puisqu'elles représentent la majorité. 
Ce n'est pas le [niveau] à l'université qui est important, mais [va-t-il] être capable de vivre en société, puis être capable de faire de quoi pour lui, d'atteindre ses rêves peu importe c'est quoi...si son rêve c'est d'être capable de marcher jusqu'au coin de la rue, bien au moins qu'il soit capable de le faire (...) ce n'est pas la profession qui est importante, c'est que la personne soit bien (...) être capable d'avoir une job au moins, d'être capable de vivre puis d'avoir peut-être une famille, même s'il [n'] en a pas de famille, puis c'est sûr qu'on regarde cela, puis [va-t-on] l'avoir à charge toute notre vie ? Puis nous autres quand on ne sera plus là il va se passer quoi ?

D'autres ont eu davantage de facilité à assumer le diagnostic posé $(n=4)$. Par ailleurs, quelques-unes soulignent que le TSA leur a permis d'évoluer comme personne, qu'il a enrichi leur vie $(n=4)$. Une mère déclare :

Avoir un enfant comme lui, honnêtement, ça nous a permis de se connaître nous en tant que personne (...) on est tous intolérants à un moment donné (...) [quand] on entend un [enfant] pleurer et faire une crise, aujourd'hui, je me dis que c'est peut-être qu'il avait quelque chose comme mon fils, donc on est plus conscientisés à ce qui nous entoure.

Difficultés au quotidien. Le diagnostic engendre des défis constants pour l'ensemble des familles. D'abord, des complications liées aux caractéristiques du TSA, telles que des rigidités alimentaires ou des troubles du sommeil, accentuent l'effet du TSA au sein de la famille. Des conséquences importantes découlant du diagnostic sont présentées dans le tableau 2.

Tableau 2. Difficultés vécues au quotidien

\begin{tabular}{lc}
\hline Difficultés au quotidien & Nombre de participantes \\
\hline Adaptations sur le plan de l'alimentation & 2 \\
Manque de sommeil & 4 \\
Effet du TSA sur la fratrie et la famille & 3 \\
Ennuis financiers & 8 \\
Difficultés liées au maintien des relations sociales & 3 \\
Difficultés conjugales & 5 \\
\hline
\end{tabular}

Le verbatim suivant représente bien la lourdeur des adaptations alimentaires qu'a dû réaliser une des participantes : « mais c'est cela, c'est dur pour la maman, car c'est beaucoup de travail (...) le lunch me prend deux heures par soir parce qu'il a des smoothies verts, des jus au juicer à chaque jour, puis cela il faut que tu le fasses, alors c'est de la job ».

Le sommeil des familles a été affecté par les difficultés de leur enfant, les paroles des mères le montrent bien : « Je ne faisais aucune nuit, je ne dormais pas la nuit. ", « La nuit on ne peut pas vraiment dormir (...) cela fait six ans que je dors 3 heures par nuit ». 
(...) le manque de sommeil, je pense que ça été mon...c'est que la chose qui m'a le plus perturbée, qui me perturbe encore même s'il a six ans (...) On a commencé par focusser sur le sommeil, c'était important que je ne sois plus une zombie, mais que je sois une personne.

Les effets du TSA sur la fratrie et la famille sont perçus dans les propos suivants : "Pour bien faire il faudrait que je sois seule avec lui. C'est difficile de s'occuper de cet enfant et de s'occuper de sa fratrie ».

C'est sûr que ce qui est difficile c'est qu'il fait beaucoup de crises et que par rapport à la fratrie, ça a beaucoup d'impact. On essaie de gérer les crises et on va céder parce qu'on a de l'énergie à mettre à d'autres places. C'est tannant ces crisettes de même...ça affecte beaucoup la fratrie.

Mais c'est sûr que les défis au quotidien sont très grands. J'ai deux autres enfants plus vieux qu'elle, donc c'est un ajustement pour toute la famille. II y a beaucoup de questionnements de la part de mes autres enfants, j'ai moins de temps pour elles (les sœurs) aussi.

Les problèmes d'argent sont importants et fréquents chez ces mères. Ils sont à la fois liés au coût du matériel et des services spécialisés que requiert leur enfant ainsi qu'à leur situation d'emploi précaire. "On investissait dans cela (les jeux qui l'intéressaient), financièrement cela peut être très coûteux ॥, " il a eu des services, c'est très dispendieux, on ne peut pas aller au Walmart acheter un jouet ».

De plus, selon les dires des participantes, ces ennuis peuvent générer des difficultés au sein de leur couple et restreindre leurs activités sociales : «On ne peut plus sortir alors on fait juste se [chicaner]...non mais quand tu n'as pas d'argent tu es [pris] et tu ne peux [plus] rien faire $»$.

Corrélativement à ce qui précède, deux mères abordent le fait que la présence du TSA nuit au maintien de leur vie sociale :

Tout le monde le sait, les gens qui se plaignent trop dérangent. Si on veut pouvoir sortir de la maison des fois et avoir une vie sociale, un semblant de vie sociale, on essaie de ne pas trop se plaindre, de ne pas trop parler de ses problèmes.

La famille est atteinte veux veux pas, parce que tout est très centré sur cet enfant-là pour éviter le plus de crises possible. Donc on ne sort pas parce qu'il n'aime pas ça, on ne va pas en vacances parce qu'il n'aime pas cela, on essaie beaucoup avec les autres enfants de les sensibiliser à la différence, mais ça reste des enfants...

Les effets du TSA sur la relation conjugale émergent également du discours des participantes : " II faut que le couple soit super solide pour passer au travers. C'est sûr que moi et mon conjoint on a failli se séparer, ça n'allait pas bien ». 
« Ça cause un stress énorme sur la vie de couple, mon mari ne connaissait pas l'autisme, donc c'était un peu difficile pour lui, il a eu de la difficulté à comprendre ».

Conciliation travail-famille. Les mères rapportent que le diagnostic nécessite une réorganisation du temps affectant leur capacité de concilier travail et famille $(n=13)$. Ainsi, le tiers des participantes révèlent qu'elles ont dû cesser de travailler pour pallier les difficultés de leur enfant, notamment leurs particularités de développement et leur besoin de soutien. Certaines ont changé d'emploi $(n=3)$ et d'autres ont diminué leurs heures de travail $(n=4)$.

Santé psychologique. Les deux tiers des mères rapportent avoir vécu des problèmes sur le plan psychologique suite au diagnostic de leur enfant. La fatigue $(n=3)$, la dépression majeure $(n=2)$, la déprime $(n=2)$, l'angoisse $(n=1)$, la tristesse $(n=1)$, la culpabilité $(n=1)$ sont les principaux problèmes nommés par les mères interrogées. Deux d'entre elles admettent avoir sollicité l'aide d'un professionnel de la santé et avoir eu recours à une médication pour surmonter leur dépression majeure.

Les données montrent que la présence du TSA de leur enfant affecte plusieurs sphères de la vie des mères concernées. La situation financière, le domaine professionnel, les relations sociales et la santé psychologique sont plus particulièrement touchés. Notons en outre que les deux mères ayant répondu aux critères cliniques de la dépression travaillaient à temps plein et éprouvaient des difficultés conjugales.

\section{Ressources personnelles}

Savoir parental. Le tiers des mères possédaient peu ou pas de connaissances sur le TSA avant le diagnostic de leur enfant. Bien qu'elles aient observé certains déficits chez leur enfant, elles ne comprenaient pas nécessairement que ceux-ci s'inséraient dans le tableau complexe du TSA. À l'inverse, d'autres mères indiquent qu'elles possédaient de bonnes connaissances sur le trouble. Une mère avait étudié dans le domaine auparavant, certaines avaient entamé des recherches sur le sujet préalablement au diagnostic $(n=7)$ et d'autres précisent que la fratrie ou un autre membre de la famille présentait également un TSA $(n=7)$. Malgré les connaissances que détenaient ces mères, leur compréhension du trouble demeure variable. Elles nomment les habiletés d'interaction et de communication sociale déficitaires, les stéréotypies, ainsi que le caractère unique, la sévérité, l'irréversibilité et la complexité du trouble. Plusieurs mères révèlent avoir reçu peu d'informations au moment du diagnostic $(n=8)$. Une d'entre elles exprime son mécontentement à ce sujet : « ils nous donnent un peu de documentation, mais on est tellement laissés à nous-mêmes... débrouillez-vous ". On nous donne le diagnostic, [mais] on n'a pas vraiment de support ». Certaines mentionnent toutefois ne pas avoir manifesté le besoin d'en savoir plus $(n=7)$. Les informations retenues correspondent aux interventions à privilégier telles que les services du CRDITED $(n=8)$, les critères diagnostiques du TSA $(n=6)$, les services offerts par le milieu hospitalier jusqu'à l'obtention de services par le CRDITED $(n=3)$, l'inscription à l'école ou à la garderie $(n=2)$, l'orthophonie ainsi que l'ergothérapie $(n=1)$ et les prestations pour personnes handicapées $(n=1)$. 
Quoique le CRDITED ait été nommé dans les interventions à privilégier, les professionnels ayant émis le diagnostic n'ont pas toujours précisé qu'il s'agirait de $I^{\prime} I C I(n=5)$ et ont encore moins expliqué le type d'intervention offert. Ainsi, leur savoir sur l'ICl était limité, et ce même après le début du traitement. Après plusieurs mois d'ICl, ces mères définissent I'ICl comme suit : une intervention intensive ciblant les sphères déficitaires du TSA pour permettre l'évolution de l'enfant $(n=9)$, une intervention basée sur l'AAC et comportant plusieurs étapes $(n=5)$, une intervention visant à outiller les parents $(n=2)$, une intervention nécessitant l'implication parentale $(n=2)$, l'intervention la plus recommandée $(n=1)$ ainsi qu'une intervention prônant l'utilisation de renforçateurs $(n=1)$. Une mère décrit l'intervention ainsi :

Ils vont toucher toutes les sphères (...) de l'enfant, de chaque structure de l'enfant. On passe par l'alimentation, la propreté, les habiletés sociales, le travail structuré (...) J'ai souvent dit que l'ICl c'était d'apprendre à apprendre à nos enfants. C'est sûr, qu'ils montrent toutes les choses qu'un enfant de deux ans doit savoir. Ça peut être au niveau des couleurs, au niveau de faire un choix, de distinguer certains éléments, donc le rôle de l'intervenant, c'est d'ouvrir un paquet de possibilités.

Certaines mères partagent leur incompréhension des plans d'intervention et soulignent le manque d'information quant aux services offerts $(n=5)$. Malgré tout, leur perception initiale de l'ICl s'avère positive $(n=12)$. De plus, bien que la plupart des mères disent avoir eu peu d'attentes envers l'ICl, elles espéraient tout de même une amélioration sur le plan du langage $(n=11)$, de l'autonomie $(n=9)$, des habiletés sociales $(n=5)$ et préscolaires $(n=5)$, des comportements $(n=4)$, de la communication $(n=4)$, de l'alimentation $(n=3)$, du sommeil $(n=2)$, des habiletés cognitives $(n=2)$ et motrices $(n=1)$.

Priorités. Les mères ont également été interrogées sur leurs attentes par rapport à leurs propres besoins. Peu de priorités spécifiques émergent quant à leur bien-être personnel, elles demeurent plutôt centrées sur celui de leur enfant ou de la famille $(n=6)$. Quelques mères soulignent même n'avoir aucun besoin individuel, qu'elles ont tendance à s'« oublier pour [leur] enfant » $(n=4)$. Une mère appuie ces propos : "Moi je passe après tout le monde, moi je donne tout ce que je peux, ce qui reste je vais le prendre. " Certaines souhaitent " survivre » $(n=1)$, diminuer leur stress $(n=1)$ et avoir plus de temps (1). Pour la famille, elles espéraient que I'ICl ait une incidence positive sur les sorties et les activités en famille $(n=2)$, la dynamique familiale $(n=2)$, la gestion des caractéristiques problématiques (ex. : sommeil, alimentation, autonomie, comportements) $(n=4)$ et une meilleure qualité de vie $(n=1)$.

Sentiment d'auto-efficacité. Les mères ont été interrogées sur leurs caractéristiques personnelles les ayant aidées à surmonter les défis engendrés par la présence du diagnostic de leur enfant. Leur discours fait émerger leurs nombreuses qualités. Elles sont proactives $(n=9)$, avocates des droits de leur enfant $(n=8)$, ouvertes d'esprit $(n=3)$, dévouées $(n=5)$ et surtout, elles démontrent d'excellentes capacités d'adaptation $(n=10)$. À cet effet, une mère se qualifiant de proactive explique : "Tu apprends aussi à être un peu la géo ou la thérapeute, tu apprends que tu ne peux pas rester assise sur ton divan à rien faire, si tu veux 
pouvoir nager la tête en haut de l'eau, pas en dessous de l'eau ! II faut que tu restes proactive, tu n'as pas le choix!»

Dans le même ordre d'idées, certaines mères ont une perception plus positive de leurs compétences parentales $(n=11)$. Elles disent avoir eu la capacité d'appliquer les tâches relatives au programme $(n=8)$ et se disent satisfaites de leur implication ( $n=5)$. Par ailleurs, deux participantes rapportent que l'ICI les a aidées à développer une meilleure confiance en soi. À l'inverse, certaines mères trouvent difficile de travailler avec leur enfant, soit par manque de collaboration de ce dernier $(n=2)$, soit parce qu'elles ne savent pas comment procéder $(n=3)$ ou qu'elles n'en voient pas les effets positifs $(n=1)$. Une mère dit que : " c'est difficile, des remises en question continuellement, la mise en doute de nos compétences de parent ». En résumé, près de la moitié des mères considèrent avoir contribué au progrès de leur enfant $(n=6)$.

Stratégies d'adaptation des mères. Les participantes ont trouvé plusieurs moyens de composer avec les difficultés découlant du diagnostic. Premièrement, la recherche d'informations est commune à la majorité d'entre elles $(n=14)$. Cette recherche visait à pallier le manque de connaissances sur le TSA et les interventions à privilégier. Pour ce faire, plusieurs médiums ont été utilisés : des moteurs de recherche tels que Google et Wikipédia $(n=11)$, des livres $(n=6)$, des formations $(n=5)$, des forums de parents $(n=3)$, des réseaux sociaux tels que Facebook $(n=2)$, des associations de parents et des fédérations (p. ex., ATEDM, Autisme Laval, TED à cœur, Fédération québécoise de l'autisme [FQA], Chat Botté) $(n=7)$. Elles avaient également recours à l'expertise des professionnels lorsqu'elles avaient des questionnements plus complexes. La plupart disent que leur recherche a été facile et utile et qu'elle les a rassurées. Une mère précise : "Ça me donne du pouvoir, empowerment ». Quelques mères déclarent avoir diminué la recherche d'informations à un certain moment, car celle-ci leur procurait plus de stress que de soulagement $(n=3)$. Deuxièmement, certaines mères ont choisi de s'impliquer dans la communauté et de « donner au suivant » $(n=3)$. Par exemple, deux mères mentionnent qu'elles siègent à des comités de parents ou à des conseils d'administration d'associations de parents. En faisant référence à sa participation au sein d'un comité, une mère affirme :

C'est ma façon à moi de donner au suivant, dans le sens de ce que je peux faire pour aider d'autres parents (...) il y a un journal qui va sortir, puis j'ai déjà écrit un article (...) je suis auteure et journaliste (...) ça me permet de parler (...) [de] tout ce qui est l'émotion des parents, je vais écrire là-dessus (...) c'est ma façon à moi de donner au suivant, je me suis beaucoup isolée, là j'essaie tranquillement de me sortir de cet isolement.

Une autre mère explique qu'elle s'est mobilisée et qu'elle s'investit dans l'accès aux services pour les parents et qu'elle vise à les informer par le biais d'ateliers qu'elle donne. Ainsi, ces mères mettent à profit leurs connaissances et partagent leur expérience pour aider des familles vivant avec un enfant présentant des besoins particuliers. Troisièmement, certaines mères indiquent que prendre du temps pour soi et avoir des projets personnels sont essentiels pour surmonter les obstacles qu'elles rencontrent $(n=4)$ : «Alors souvent je vois que c'est un 
manque d'énergie, un manque de repos alors (...) je m'arrête un peu, j'essaie de prendre du temps pour moi, [pour] me recharger, c'est surtout ça ». Des voyages en amoureux, des cours de yoga, la planification d'un mariage, l'écriture et la relaxation figurent parmi les moyens choisis permettant à la « batterie de se recharger pour continuer ». Quatrièmement, tel que mentionné précédemment, la conciliation travail-famille s'avère ardue. Ainsi, comme solution, plusieurs mères ont effectué des changements relatifs à leur emploi, tels que la diminution du nombre d'heures $(n=5)$, l'arrêt de travail $(n=2)$ ou une réorientation $(n=5)$. Cela leur a permis de mieux répondre aux besoins spécifiques de leur enfant et de réaliser les tâches relatives à son éducation ainsi que les tâches domestiques au quotidien. Cinquièmement, certaines mères se sont plutôt investies autrement dans l'intervention, soit par l'achat ou la création de matériel de thérapie $(n=3)$ ou bien par l'organisation de rencontres avec l'ensemble des intervenants de leur enfant $(n=1)$.

Implication parentale. L'implication parentale dans l'ICI se manifeste sous différentes formes. Dans le cadre de cette étude, six ont été relevées : la présence aux séances d'ICl, la généralisation et le maintien des acquis, la cotation dans le cartable d'intervention, l'intervention à titre de cothérapeute, la communication avec les éducatrices et la participation aux supervisions. D'abord, certains des milieux offraient aux mères d'assister aux séances d'intervention $(n=8)$. Leur participation était variable. La moitié d'entre elles préféraient ne pas y être présentes $(n=5)$, soit qu'elles n'en ressentaient pas le besoin, soit que cela pouvait distraire leur enfant ou qu'elles avaient besoin de répit. Une mère exprime son mécontentement à l'égard du fait qu'on ne lui ait pas offert :

À un moment donné, j'ai dit « regardez, moi je veux apprendre comment travailler avec mon fils, [puis-je] assister ? " "Ah bien oui, tu peux venir ! » Mais on ne me l'a jamais offert. Donc pendant neuf mois, je n'ai jamais assisté parce que personne ne m'a offert d'y aller.

Celles qui y allaient le faisaient rarement $(n=4)$ ou régulièrement $(n=2)$. Une mère explique les raisons pour lesquelles elle préférait ne pas y assister:

J'ai décidé de me distancer un peu, pour me donner aussi un petit répit, mais aussi pour que mon enfant apprenne aussi à recevoir l'apprentissage de quelqu'un d'autre sans que je sois tout le temps là à le tenir par la main ou derrière, cachée derrière une vitre, pour qu'il apprenne aussi que je ne peux pas tout le temps être collée sur lui.

Ensuite, les mères étaient invitées à poursuivre les interventions auprès de leur enfant à domicile. Certaines mères appliquaient des interventions reliées aux comportements difficiles $(n=2)$ et à l'autonomie $(n=3)$, mais, pour la plupart, leur participation se centrait sur la généralisation et sur le maintien des acquis $(n=14)$. Trois des mères avaient accès au cartable d'intervention et une seule d'entre elles effectuait la cotation des interventions. Une des mères précise même qu'on lui refusait le droit de le consulter : " le cartable il a fallu que je me batte, elle n'avait pas le droit de me le donner...elle n'avait pas le droit de me le laisser ». Trois des répondantes disent avoir joué le rôle de cothérapeute dans l'intervention, sans toutefois avoir effectué de séances structurées. Deux des mères contribuaient à l'intervention en 


\section{8}

préparant le matériel nécessaire et en fournissant les renforçateurs. La presque totalité des mères se présentaient toujours aux supervisions lorsqu'elles y étaient invitées $(n=13)$. Leur participation à ces rencontres était pour la plupart volontaire $(n=12)$ et parfois obligatoire $(n=3)$. La plupart d'entre elles trouvaient ces rencontres utiles $(n=8)$. Une des mères partage sa compréhension quant à l'importance de son implication : "Un parent impliqué, ils m'ont dit que ça fait une différence pour l'enfant (...) un parent qui s'implique, oui, ce n'est pas juste les autres, c'est une équipe ».

Finalement, plus de la moitié des répondantes qualifient la communication avec les éducatrices de fréquente ou quotidienne $(n=8)$. Elle pouvait se faire en personne, par téléphone, par le biais d'un cahier de liaison ou par courrier électronique. Une des mères ayant eu plus de difficulté à obtenir de l'information de la part de son éducatrice indique qu'elle devait poser « un million de questions (...) j'essayais d'avoir du feedback, je n'en avais pas. »

Cinq mères rapportent qu'elles n'intervenaient pas auprès de leur enfant, soit parce qu'elles désiraient se « dégager » de l'intervention ou qu'elles n'avaient pas été encouragées à s'impliquer :

Moi je ne voulais pas faire la thérapie à la maison (...) je voulais me dégager de ça. Ce n'était pas un chapeau que je voulais porter (...) On dirait que j'ai eu à préserver aussi mon rôle de maman là-dedans. Je disais que moi je m'occupais de la sécurité affective puis que oui j'étais toujours disponible pour donner des idées et que pour faire avancer plein de choses, mais on a voulu se décharger aussi quelque part.

« Non, la superviseure m'a dit vous êtes maman, pas intervenante vous êtes une maman, c'est la job de maman ». Les mères ont été questionnées sur leur degré de satisfaction quant à leur implication parentale. La plupart sont satisfaites de leur implication $(n=8)$, mais elles mentionnent tout de même certains aspects qui auraient pu contribuer à son amélioration. Elles nomment l'offre d'assister aux séances structurées $(n=2)$, la précocité de l'intervention $(n=2)$, une meilleure coopération de l'enfant $(n=1)$, l'accès aux formations $(n=1)$ et une implication plus grande du père de l'enfant $(n=1)$. Inversement, parmi les facteurs ayant pu freiner leur implication, elles mentionnent le manque de temps $(n=1)$, l'absence de résultats positifs de l'intervention $(n=1)$, l'exclusion de leurs besoins $(n=1)$ ainsi que le manque d'ouverture et de disponibilités des éducatrices $(n=1)$. Quatre répondantes indiquent que rien n'aurait pu influencer leur degré d'implication.

Les ressources personnelles que détiennent les mères d'enfant présentant un TSA apparaissent comme des facteurs facilitant leur expérience. Ces mères sont dotées de connaissances par rapport au TSA et à I'ICl, elles possèdent d'excellentes stratégies ainsi qu'une perception positive d'elle-même et de leur sentiment d'auto-efficacité. Leurs priorités sont bien claires, elles désirent plus que tout, l'évolution de leur enfant. Pour ce faire, elles s'investissent davantage et semblent même oublier leurs propres besoins. Les données suggèrent que la mobilisation de l'ensemble de leurs ressources personnelles leur ait permis de composer avec les défis rencontrés et les lacunes observées. II est possible 
d'identifier certains éléments ayant pu nuire à leur expérience. Les limites sur le plan de la transmission de l'information auraient pu affecter leur compréhension du diagnostic et de l'intervention, en plus d'influencer leur degré d'implication parentale. Cependant, leur proactivité leur a permis de recueillir les informations dont elles avaient besoin. Finalement, la communication entre ces mères et les éducatrices n'était pas toujours fluide.

\section{Soutien}

Selon les mères interrogées, plusieurs formes de soutien ont été présentes en contexte d'ICl, alors que d'autres ont été absentes. D'abord, les principaux types de soutien discutés sont : social, émotif, récréatif, instrumental et informatif. Les professionnels (pédopsychiatre, médecin, psychologue, ergothérapeute, orthophoniste, travailleuse sociale), particulièrement les éducatrices, les services de répit, les associations de parents ainsi que les amis et la famille sont les principales sources de soutien nommées.

Soutien social. La majorité des mères rapportent avoir eu peu de soutien social $(n=10)$. Elles expliquent qu'elles ont eu de la difficulté à maintenir des relations harmonieuses avec leur famille et leurs amis en raison du temps insuffisant, de la distance, mais surtout du manque de compréhension de leur entourage : "Quand tu as des enfants handicapés, on dirait que les amies disparaissent » et « la famille n'est pas de soutien non plus ». Par conséquent, elles se sont isolées $(n=4)$ ou se sont dirigées vers les ressources communautaires $(n=6)$. Elles rapportent avoir davantage de facilité à créer des liens avec des familles d'enfant ayant des besoins particuliers $(n=4)$.

Soutien émotif. Le soutien émotif perçu est variable. Le tiers des mères disent en avoir manqué. Ces mères rapportent que le soutien émotif de la part de la famille et des amis était peu présent. De plus, bien que certaines mères aient bénéficié des services de travailleuses sociales, deux d'entre elles précisent que cela a été peu utile. Une répondante suggère qu'il serait bénéfique d'offrir des services en psychologie aux mères d'enfant présentant un TSA à la suite de l'annonce du diagnostic : " on pleure, on pleure notre peine (...) on pourrait nous référer à un psychologue, pour parler, pour trouver des solutions ». Certaines répondantes affirment avoir reçu du soutien informel de leur conjoint $(n=4)$, de leur famille $(n=7)$, de leurs amis $(n=8)$ ou du soutien formel des éducatrices $(n=6)$, des superviseures $(n=2)$, des travailleuses sociales $(n=1)$ et des pédopsychiatres $(n=1)$ intervenant auprès de leur enfant : " Ma psychoéducatrice de l'ICl elle a toujours été là pour me soutenir, pour me conseiller, j'ai même pleuré des fois ». Malgré la présence de ce type de soutien, deux mères mentionnent qu'elles désiraient épargner leurs proches et leurs amis et, de ce fait, elles faisaient rarement appel à leur entourage. Deux mères avaient également de la difficulté à se confier aux éducatrices de l'enfant parce qu'elles considéraient que cela ne faisait pas partie intégrante de leur mandat ou parce qu'elles les jugeaient trop jeunes et inexpérimentées pour pouvoir les soutenir. Deux mères disent ne pas avoir eu de besoin émotif particulier.

Soutien instrumental. La majorité des mères vivent des difficultés financières en raison des frais associés au TSA de leur enfant et des changements 
relatifs à leur emploi $(n=12)$. D'ailleurs, elles expriment clairement leur besoin : « financièrement, en fait c'est un problème que j'estime financièrement...le soutien qui a le plus manqué et qui manque encore, c'est le soutien au quotidien ». Certaines d'entre elles reçoivent des subventions du Centre local de services communautaires $(C L S C)(n=5)$ ou une allocation pour enfants handicapés $(n=7)$. Le cinquième des répondantes a bénéficié de subventions de fondations pour enfants en difficulté. Par ailleurs, quelques mères déplorent les conditions d'admissibilité restreintes à ces bourses. Deux répondantes ont eu recours à des ressources communautaires pour obtenir du matériel de thérapie, des jeux et des vêtements. Les éducatrices travaillant au CRDITED ainsi que le comité des usagers des CRDITED pouvaient également offrir une aide matérielle $(n=4)$. De plus, quelques-unes des participantes rapportent qu'elles recevaient une contribution financière ou matérielle d'un membre de leur famille $(n=4)$.

Certaines mères $(n=7)$ affirment avoir eu besoin d'aide sur le plan de leurs responsabilités parentales, par exemple, pour la réalisation de leurs tâches quotidiennes, pour la gestion du transport de leur enfant, pour remplir divers formulaires ou encore pour garder leurs enfants : « Aide-moi en mettant quelqu'un qui fait mon ménage chez moi tous les jours...faire la bouffe pendant que moi je m'occupe de mon fils, mais je ne peux pas tout faire. Je suis toute seule, je ne peux pas ». D'abord, deux mères rapportent que le grand-père de l'enfant s'est chargé des déplacements lors de l'intervention. Parallèlement, une mère révèle que son éducatrice lui offrait, à l'occasion, de s'occuper du transport de l'enfant. Deux mères ont obtenu du soutien de leur travailleuse sociale ou de leur pédopsychiatre pour remplir les documents relatifs à la demande d'allocation pour enfants handicapés. À l'inverse, une des participantes se plaint de ne pas avoir reçu davantage d'aide pour effectuer la demande de subvention pour le transport. Une autre mentionne que sa ressource communautaire lui a offert un service à domicile pour effectuer les tâches ménagères : "Les ressources communautaires m'ont aidée, sont venues chez nous. Ils ont fait du ménage quand ils sont venus (...) ça c'était très apprécié ". Une des mères dit aussi avoir été soutenue par sa belle-mère qui l'accompagnait à ses rendez-vous dans les moments les plus difficiles. Une mère se considère chanceuse, car son employeur l'a soutenue dans ses démarches en lui offrant une flexibilité au travail pour lui permettre de se rendre à ses nombreux rendez-vous. D'autres mères ont bénéficié de gardiennage par les grands-parents et par les ressources communautaires $(n=2)$. Par ailleurs, le besoin de répit s'avère important pour " garder l'équilibre de la famille ". Les mères rapportent toutefois que les services de répit sont dispendieux et qu'ils ne parviennent pas toujours à prendre en charge les enfants, surtout si plus d'un présente une difficulté. Ainsi, une des mères admet être réticente à y envoyer son enfant, elle souhaiterait plutôt qu'une intervenante vienne à domicile. Malgré tout, certaines disent en profiter occasionnellement et apprécier : "À tous les mois, on a une fin semaine de répit. Ou aux deux mois. Juste pour faire des activités. »

Soutien récréatif. Seules deux participantes indiquent avoir reçu du soutien récréatif pendant l'intervention. L'une d'entre elles s'est vue offrir gratuitement des cours de danse et l'autre était, à l'occasion, invitée à des fêtes organisées par sa ressource communautaire qui regroupaient des parents d'enfants différents. Elles disent avoir apprécié ces gestes qui leur permettaient d'avoir un peu de répit. 
Les autres mères n'ont pas bénéficié de soutien récréatif. Une d'elles mentionne toutefois qu'une éducatrice lui avait offert de l'accompagnement pour les activités de l'enfant, ce qu'elle a perçu comme étant du soutien récréatif.

Soutien informatif. Comme mentionné précédemment, le besoin de s'informer est très présent chez les mères interrogées. Nombreuses sont celles déclarant avoir manqué de cette forme de soutien. Le tableau 3 présente les sources desquelles elles ont obtenu des informations.

Tableau 3. Sources de soutien informatif

\begin{tabular}{lc}
\hline Sources de & $\begin{array}{c}\text { Nombre de } \\
\text { soutien informatif }\end{array}$ \\
\hline Formel & 1 \\
Office des Personnes
\end{tabular}

Les informations reçues varient : les méthodes d'intervention ( $n=7)$, le TSA et son évolution $(n=3)$, la gestion des crises $(n=3)$, les troubles associés $(n=2)$, les allocations pour personnes handicapées $(n=2)$, les droits de l'enfant et de sa famille $(n=1)$ et l'utilisation de pictogrammes $(n=1)$. Les informations perçues comme les plus utiles sont celles liées aux prestations pour personnes handicapées et celles liées aux techniques d'intervention. Certaines mères disent avoir bénéficié de brèves activités d'apprentissage offertes gratuitement par le CRDITED $(n=7)$ sur le TSA $(n=3)$, sur l'ICI $(n=3)$ et sur d'autres méthodes d'intervention inspirées de TEACCH (Treatment and Education of Autistic and related Communication handicapped Children) $(\mathrm{n}=2)$ et de PECS (Picture Exchange Communication System) $(\mathrm{n}=2)$. Ces ateliers pouvaient avoir lieu avant ou après le début des services, parfois même vers la fin de l'ICl. D'autres ont également reçu des formations plus spécifiques sur les besoins sensoriels $(n=1)$, sur les scénarios sociaux $(n=1)$ et sur la fratrie $(n=1)$. Quatre participantes les ont trouvées utiles. Une des participantes critique toutefois le format très théorique et peu pratique de celles-ci. Une mère mentionne qu'elle aurait apprécié un suivi avec les formateurs. Près de la moitié des répondantes se sont inscrites à des formations plus approfondies afin d'acquérir de nouvelles 
connaissances sur la condition de leur enfant et sur les moyens d'intervention. Ainsi, elles ont assisté à des présentations sur l'ICI, l'AAC, le TSA, les émotions, TEACCH, PECS et l'utilisation du iPad. Celles-ci étaient données par des superviseurs en clinique privée, des orthophonistes affiliées à des commissions scolaires ainsi qu'une mère d'enfant présentant un TSA et une personne ayant un TSA. Chacune des formations était perçue positivement. Une des répondantes ayant suivi un programme universitaire sur l'ICI avant la naissance de son enfant, parce qu'elle travaillait en adaptation scolaire, rapporte que cela lui a été très bénéfique.

Relations avec les éducatrices. La majorité des mères qualifient leur relation avec les éducatrices de leur enfant comme bonne, très bonne ou excellente $(n=14)$. Elles soulignent l'importance de la qualité de cette relation dans l'intervention : " Je pense que c'est important qu'on ait un semblant de relation d'amitié. Premièrement, elles nous aident beaucoup, elles aident beaucoup nos enfants. Si on n'est pas à l'aise avec cette personne qui s'occupe de notre enfant, je ne crois pas que ça aurait été aussi bien pour l'enfant. » Elles décrivent leur relation comme sincère, amicale, respectueuse et empreinte de confiance. Les qualités qui ont été les plus appréciées chez l'éducatrice sont : l'écoute $(n=5)$, le dévouement $(n=3)$, la gentillesse $(n=3)$, la douceur $(n=2)$, l'empathie $(n=2)$, l'ouverture d'esprit $(n=2)$, la compétence $(n=2)$, les conseils $(n=2)$, la générosité $(n=2)$, la patience $(n=2)$, la collaboration $(n=1)$ et le dynamisme $(n=1)$. « Elle était fine, elle était fine cette fillelà, elle était douce, fine, attentionnée, ahhhh de la soie, vraiment super fine, mais vraiment vraiment gentille. Donc la dernière journée, on pleurait ». Certains facteurs tels que la permanence, la créativité, l'autorité, l'âge, la formation, l'expérience et le fait d'être parent influencent également la perception qu'elles ont des éducatrices. Une seule mère rapporte avoir eu une relation plus amère avec une des éducatrices. Elle avait peu confiance en elle et elle la considérait intrusive :

La dernière année, elle voulait me cuisiner, elle jouait le rôle du psychologue, jusqu'à temps que je lui dise : « ce n'est pas de tes affaires comment je me sens ». Elle est jeune et il faut commencer quelque part, tout le monde commence quelque part, mais à 19 ans tout frais tu viens de sortir du cégep, tu as fait une technique, pas de préjugés, mais tu n'as pas l'expérience pour faire la morale non plus, ça a bien commencé la confiance était bonne, pour ne plus avoir de confiance, j'ai trop d'amertume face à certains intervenants, beaucoup de méfiance.

Les éducatrices jouent ainsi un rôle central dans l'intervention. Comme mentionné précédemment, elles offrent divers types de soutien : émotif, instrumental et informatif. À cela s'ajoute aussi le coaching. Le tiers des mères disent en avoir bénéficié. Les éducatrices peuvent faire des démonstrations lors des rencontres de supervision ou encore, elles se déplacent à domicile pour les aider à comprendre, à implanter et à appliquer une intervention : « on m'expliquait une technique, comment faire la demande (...) comment faire l'échange d'information ».

Les répondantes ont été interrogées sur les bons et les moins bons moments vécus avec les éducatrices. Les réussites de l'enfant $(n=3)$, l'appréciation de l'enfant $(n=3)$ et les fêtes $(n=1)$ figurent parmi les nombreux bons moments partagés avec elles. «C'est de la voir, il était attaché à elle. C'était de la voir avec lui. On voyait 
qu'il y avait une complicité entre les deux, c'était beau de les voir ". Les absences fréquentes $(n=6)$, le taux de roulement élevé $(n=3)$, les oublis des éducatrices $(n=2)$ et trouver des personnes qualifiées $(n=1)$ sont perçus comme les éléments les plus difficiles de l'intervention. "Puis de travailler aussi avec eux puis c'est pas facile de trouver les bonnes personnes pour travailler avec ton enfant ", "Mais je vais quand même te dire ce que je trouve déplorable, c'est que ce ne soit pas toujours la même éducatrice qui s'occupe de notre enfant (...) puis je trouve qu'elle manquait souvent ».

Certes, le soutien constitue un facteur de protection pour les mères, toutefois peu d'entre elles en ont bénéficié. Les mères identifient clairement ce qui a été utile pour elles et ce dont elles ont besoin pour améliorer leur réalité quotidienne. À maintes reprises, les éducatrices sont nommées. Elles sont présentées comme une source de soutien social, informatif et émotif important. Les mères apprécient particulièrement le soutien financier et l'aide pour s'acquitter de certaines de leurs responsabilités parentales. Finalement, il semble que certaines mères aient développé davantage de moyens pour accéder aux services de soutien offerts aux familles d'enfant présentant un TSA. Encore ici, il est possible que leur capacité d'initiative les ait aidées dans leur recherche d'information, de ressources et de formation.

\section{Discussion}

L'objectif de cette étude était de recueillir des données sur l'expérience des mères d'enfants présentant un TSA ayant bénéficié d'ICI. Les entrevues réalisées ont permis d'explorer les défis rencontrés depuis l'obtention du diagnostic de leur enfant, leurs ressources personnelles ainsi que le soutien dont elles ont bénéficié et qui leur ont permis de composer avec les difficultés vécues.

II en ressort que les défis observés chez les mères d'enfant présentant un TSA sont comparables à ceux documentés dans les écrits. D'abord, l'annonce d'un diagnostic est souvent vécue difficilement par les mères. En comparant les données de cette étude avec celles de Poirier (2008), on constate des similitudes en ce qui a trait aux réactions des mères. Elles sont choquées par la nouvelle et s'inquiètent pour l'avenir de leur enfant. Néanmoins, la recherche actuelle met en évidence un fait intéressant, le TSA aurait permis de sensibiliser certaines mères aux difficultés auxquelles peuvent faire face d'autres familles. Ensuite, les résultats montrent que le TSA engendre des difficultés importantes au sein de la famille ainsi que des problèmes de santé physique chez la personne atteinte du trouble. Le bien-être et la qualité de vie de ces familles sont donc altérés. Effectivement, de nombreuses études ont démontré que les parents d'enfants présentant un TSA éprouvent des difficultés conciliation travail-famille, des problèmes financiers et des troubles de santé psychologiques à la suite du diagnostic de leur enfant (Allik et al., 2006; Eisenhower et al., 2005; Giarelli et al., 2005; Montes \& Halterman, 2007; Sénéchal \& des Rivières-Pigeon, 2009; Twoy et al., 2007; Wintgens \& Hayez, 2006). De plus, la littérature indique que les personnes ayant un TSA sont plus à risque de développer des problèmes liés à l'alimentation (Bandini et al., 2010; Martins et al., 2008) et au sommeil (Cotton \& Richdale, 2006). L'étude révèle également que la présence du TSA peut nuire à la fratrie et à la qualité des relations sociales et 
de couple. Bien que les effets des symptômes autistiques aient été étudiés dans le contexte des relations sociales et conjugales (Courcy \& des Rivières-Pigeon, 2012), peu d'auteurs se sont intéressés à l'expérience de la fratrie.

En ce qui a trait aux ressources personnelles des parents, les mères qui détenaient de l'information sur le TSA avant l'annonce du diagnostic ont pour la plupart effectué des recherches afin de vérifier leurs soupçons, ce qui est semblable aux données de l'étude de Poirier et Goupil (2008). Même si peu d'informations leur ont été transmises sur le TSA ou l'ICl lors de la rencontre diagnostique, les mères semblent ne pas avoir manifesté le besoin d'en savoir davantage. L'information la plus retenue au moment de la rencontre diagnostique est l'offre de services des CRDITED. Ainsi, leur compréhension de l'ICl se limitait au fait que cette approche est une intervention intensive visant à améliorer les habiletés déficitaires de l'enfant se basant sur les principes de l'AAC. Somme toute, leur perception de I'ICI à ce moment-là était positive et elles espéraient des progrès, notamment sur le plan du langage et de l'autonomie. II est intéressant de noter que leurs priorités pendant l'intervention étaient presque toujours centrées sur les besoins de leur enfant plutôt que sur leur bien-être personnel. Ensuite, les données de l'étude suggèrent que les mères possèdent d'excellentes capacités d'adaptation, des qualités et un sentiment d'auto-efficacité qui leur ont permis de surmonter les défis rencontrés. II est possible que la perception positive de leurs compétences parentales ait favorisé leur implication, principalement en ce qui a trait à la généralisation et au maintien des acquis, tel que le supposent certains auteurs (Solish \& Perry, 2008).

L'étude révèle diverses stratégies d'adaptation utilisées par les mères. Leur principal moyen est la recherche d'informations, plus spécifiquement, par le biais d'internet, des associations de parents et des fédérations. Ces mères indiquent que cela a pour effet de les sécuriser. Cette façon de contrer la méconnaissance correspond à une des stratégies identifiées par Veyssière et ses collaborateurs (2010). Ensuite, les remaniements relatifs à l'emploi ont un effet non négligeable dans l'expérience de ces mères. Finalement, quelques-unes $(n=3)$ soulignent l'importance de donner au suivant, de s'impliquer dans l'intervention, d'avoir du temps pour soi ainsi que des projets personnels liés ou non à la problématique de leur enfant.

Par ailleurs, les formes d'implication parentale émergeant de cette étude ont été comparées avec la littérature sur le sujet (Granger et al., 2010; Solish \& Perry, 2008). Solish et Perry (2008) avaient identifié six tâches que pouvaient effectuer les parents dans le cadre de l'intervention : 1) la communication avec les intervenants, 2) la participation aux supervisions, 3) les discussions concernant le contenu du programme 4) les séances structurées d'ICI, 5) la généralisation des acquis ainsi que 6) la gestion des comportements inappropriés. Des similitudes s'observent quant à la manière de s'impliquer dans l'intervention. Par ailleurs, la généralisation et le maintien des acquis, la communication avec les éducatrices et la participation aux supervisions sont celles qui prédominent au sein de l'échantillon. Les autres formes d'implication, telles que la participation aux séances structurées, la cothérapie et la cotation étaient peu présentes et de toute façon peu encouragées par le milieu et par les éducatrices intervenant auprès de l'enfant, alors que les études sont nombreuses à faire preuve de l'importance d'inclure la famille dans 
l'intervention (Chrétien et al., 2003; Guralnick, 2000, 2001, 2005; National Research Council Committee on Educational Interventions for Children with Autism, 2001). Rappelons également que le programme de Lovaas recommande l'implication des parents à titre de cothérapeute auprès de leur enfant plusieurs heures par semaine (Lovaas, 1987).

Plusieurs lacunes s'observent sur le plan des soutiens social, émotif, informatif et instrumental que reçoivent les mères d'enfant présentant un TSA. D'abord, en ce qui concerne le soutien social, elles ont tendance à s'isoler ou à créer des liens avec des personnes vivant des situations semblables. Par le fait même, certaines n'ont pas bénéficié de soutien émotif. Les membres de la famille, les amis et les éducatrices demeurent les principales personnes sur lesquelles elles peuvent compter et à qui elles peuvent se confier. Les données recueillies sont similaires à celles d'une étude récente examinant les déterminants sociaux de la santé et des symptômes dépressifs chez les mères d'enfants ayant un TSA qui concluent à un besoin de soutien important. Plus spécifiquement, près de la moitié des mères rapportent avoir manqué de soutien social, instrumental et récréatif (Courcy \& des Rivières-Pigeon, 2013). Les résultats de l'étude révèlent que les mères estiment avoir reçu peu de soutien informatif notamment en ce qui a trait aux méthodes d'intervention. II semble toutefois que les éducatrices de leur enfant soient une source considérable d'informations. La documentation concernant les prestations offertes aux personnes handicapées leur a été très utile. Parallèlement, plusieurs études ont démontré l'importance de former les mères et de les soutenir dans leurs interventions, et ce, dès le début de l'ICl afin de favoriser l'implication parentale, la généralisation et le maintien des acquis (Granger et al., 2010; Hayward et al., 2009; Lovaas, 1987). Cependant, peu de mères de l'étude ont reçu une formation spécifique sur l'ICl et bénéficié de coaching. Ces lacunes ont probablement eu une incidence négative sur leur degré d'implication et, par le fait même, sur la réussite de l'intervention. La participation à des formations offertes par les organismes privés est toutefois révélatrice de leur désir d'approfondir leurs connaissances sur la multitude d'interventions et les spécificités du TSA. Une seule mère possède une formation universitaire reliée à la problématique de son enfant. On conviendra ici de la pertinence de diriger les mères vers des formations basées sur des résultats probants afin d'aider ces mères à mieux comprendre le trouble de leur enfant et à intervenir plus efficacement. En ce qui a trait au soutien instrumental, certaines mères bénéficient des prestations pour personnes handicapées. Les résultats de l'étude mettent en lumière l'importance d'une aide liée à l'ensemble de leurs responsabilités parentales. Les données indiquent que le soutien reçu, aussi rare soit-il, les a aidées à préserver une certaine qualité de vie. Toutefois, compte tenu du manque de soutien disponible, il est impossible d'observer les effets positifs du soutien sur la diminution du stress chez les mères, tels que documentés (Dunn et al., 2001; Montes \& Halterman, 2007; Siklos \& Kerns, 2006; Twoy et al., 2007; White \& Hastings, 2004).

Tel que mentionné précédemment, les éducatrices constituent une source de soutien importante pour les mères. Puisque leurs tâches dépassent la réalisation de l'intervention auprès de l'enfant, leur rôle est central dans l'ICl. À cet égard, le soutien et l'implication de l'équipe d'intervention ont été identifiés comme des variables favorisant le développement de l'enfant (Beaud \& Quentel, 


\section{6}

2011a) et l'implication parentale (Sabourin et al., 2011). Par contre, le haut taux de roulement et les absences fréquentes des éducatrices ont été moins appréciés. Ces facteurs aussi soulevés dans une étude sur la fidélité de l'implantation peuvent être associés à la qualité de l'intervention (Gamache et al., 2011). Ils peuvent également influencer la perception des parents à l'égard de I'ICI. Par ailleurs, certains auteurs ont révélé que la perception des parents peut être associée à leur implication et, par conséquent, à l'efficacité de l'intervention (Blacklock et al., 2012; Solish \& Perry, 2008). Pour toutes ces raisons, il appert essentiel d'examiner de plus près les variables pouvant expliquer l'instabilité du personnel afin de développer une organisation favorisant leur rétention et leur assiduité au travail.

Les résultats et les conclusions de cette étude qualitative exploratoire doivent être interprétés en tenant compte de ses contraintes méthodologiques. La petite taille de l'échantillon, les caractéristiques distinctes des enfants ainsi que l'homogénéité du profil des participantes ne permettent pas de représenter l'ensemble des mères d'enfant présentant un TSA et ayant reçu de l'ICI et surtout de généraliser les résultats. Compte tenu des difficultés découlant du diagnostic, il est possible que les mères ayant répondu à la demande de participation à cette étude soient des mères éprouvant des difficultés moindres ou détenant des ressources personnelles remarquables.

\section{Conclusion}

En somme, le présent article permet de documenter l'expérience des mères d'enfant présentant un TSA ayant reçu une $\mathrm{ICI}$, notamment en ce qui a trait aux obstacles découlant du diagnostic, à la mobilisation de leurs ressources personnelles, aux stratégies d'adaptation qu'elles perçoivent déployer ainsi qu'au soutien qu'elles ont reçu. D'abord, il semble bien que leurs ressources personnelles et leur sentiment d'auto-efficacité leur permettent de se mobiliser et de s'engager dans le processus. Ensuite, elles développent des moyens pour s'adapter aux difficultés qu'elles vivent au quotidien. De plus, elles sont constamment à la recherche de soutien de quelque nature que ce soit, aussi petit soit-il, pour les aider à composer avec les obstacles engendrés par le diagnostic de TSA. Les lacunes sur le plan des informations transmises quant au TSA et à l'ICI, sur le plan des formations, sur le plan de l'inclusion de la famille dans l'intervention et sur le plan du soutien se perçoivent et, par conséquent, nuisent à leur compréhension du trouble et de l'intervention. Cela soulève un besoin important quant au développement et à l'amélioration des services offerts aux familles d'enfant présentant un TSA, mais également quant aux recherches à effectuer dans ce domaine. D'abord, la bonification des services offerts aux familles principalement sous la forme d'un soutien financier, informatif et émotif plus substantiel pourrait contribuer à la diminution des défis auxquels elles font face, au développement de leurs connaissances, de leurs pratiques d'intervention et de leur implication parentale en plus d'améliorer leur bien-être et leur qualité de vie. Ces changements dans l'offre de services représenteraient de grands avantages pour les enfants ayant un TSA qui connaîtront de ce fait une évolution plus grande et pourront réaliser leur plein potentiel grâce aux ressources améliorées dont disposeront leurs parents. En ce qui a trait à la recherche, des études auprès de plus grands échantillons s'imposent et l'inclusion des pères serait également plus que souhaitable afin de 
mieux représenter la réalité de ces familles. L'élaboration et la validation d'outils pour mesurer de manière quantitative les informations que détiennent les parents au sujet du TSA et de l'ICI semblent essentielles au développement d'une meilleure compréhension. Cela servirait également à connaitre les informations dont ont besoin les parents et permettrait de concevoir un modèle de soutien pour les accompagner dans la démarche d'intervention et ainsi permettre l'actualisation du potentiel de l'enfant. De plus, les stratégies employées par les mères gagneraient à être approfondies. Les mères interrogées ont certes des capacités d'adaptation leur ayant permis de surmonter les obstacles présents, cependant ce ne sont pas tous les parents d'enfant présentant un TSA qui s'adaptent ainsi. II s'avère donc essentiel de poursuivre les recherches concernant les stratégies d'adaptation en rejoignant un plus grand nombre de parents afin d'identifier d'autres moyens employés et de vérifier s'il existe des distinctions dans la façon dont des parents ayant un profil différent s'ajuste dans de telles circonstances. Cela permettrait également de développer des services et d'offrir du soutien pouvant davantage répondre aux besoins de parents, quelles que soient leurs caractéristiques.

\section{Références}

Allik, H., Larsson, J. O., \& Smedje, H. (2006). Health-related quality of life in parents of school-age children with Asperger syndrome or high-functioning autism. Health and Quality of Life Outcomes, 4(1), 1-8.

American Psychiatric Association (2013). Diagnostic and statistical manual of mental disorders (5th ed.). Washington, DC.

Anderson, K. J., \& Minke, K. M. (2007). Parent involvement in education: Toward an understanding of parents' decision making. The Journal of Educational Research, 100(5), 311-323.

Bandini, L. G., Anderson, S. E., Curtin, C., Cermak, S., Evans, E. W., Scampini, R., . . Must, A. (2010). Food selectivity in children with autism spectrum disorders and typically developing children. The Journal of Pediatrics, 157(2), 259-264.

Bandura, A. (1977). Self-efficacy: toward a unifying theory of behavioral change. Psychological Review, 84(2), 191-215.

Bandura, A. (1989). Human agency in social cognitive theory. American Psychologist, 44(9), 1175-1184.

Beaud, L., \& Quentel, J.-C. (2011a). Information et vécu parental du diagnostic de l'autisme. I. Premières identifications et nature des premières inquiétudes.
Annales Médico-psychologiques, revue psychiatrique, 169(1), 54-62.

Beaud, L., \& Quentel, J. C. (2011b). Information et vécu parental du diagnostic de l'autisme II. Effets des troubles et qualité de vie. Annales médicopsychologiques, Revue psychiatrique, 169(2), 132-139.

Benson, B., \& Dewey, D. (2008). Parental stress and needs in families of children with autism spectrum disorder. International Journal of Disability, Community \& Rehabilitation, 7(1). Repéré à http://www.ijdcr.ca/ VOL07_01_CAN/articles/benson.shtml.

Benson, P., Karlof, K. L., \& Siperstein, G. N. (2008). Maternal involvement in the education of young children with autism spectrum disorders. Autism, 12(1), 4763.

Blacklock, K., Weiss, O., Perry, A., \& Freeman, N. (2012). Brief Report: Parents' perception of progress versus children's actual progress in intensive behavioural intervention. Journal on Developmental Disabilities, 18(3).

Bowker, A., D'Angelo, N. M., Hicks, R., \& Wells, K. (2011). Treatments for autism: Parental choices and perceptions of change. Journal of Autism and Developmental Disorders, 41(10), 13731382. 
Bromley, J., Hare, D. J., Davison, K., \& Emerson, E. (2004). Mothers supporting children with autistic spectrum disorders. Autism, 8(4), 409.

California Departments of Education (1997). Best practices for designing and delivering effective programs for individuals with autistic spectrum disorders: Recommendation of the Collaborative Workshop on Autism Spectrum Disorders. Sacramento, CA: Author.

Cappe, E., Wolff, M., Bobet, R., \& Adrien, J. L. (2011). Quality of life: a key variable to consider in the evaluation of adjustment in parents of children with autism spectrum disorders and in the development of relevant support and assistance programmes. Quality of Life Research, 1-16.

Centers for Disease Control Prevention. (2012). New data on autism spectrum disorder. Repéré à http://www.cdc.gov/ features/countingautism/.

Chrétien, M., Connolly, P., \& Moxness, K. (2003). Trouble envahissant du développement : un modèle d'intervention précoce centrée sur la famille. Santé mentale au Québec, 28(1), 151-168.

Cohen, H., Amerine-Dickens, M., \& Smith, T. (2006). Early intensive behavioral treatment: Replication of the UCLA model in a community setting. Journal of Developmental \& Behavioral Pediatrics, 27(2), 145-155.

Cotton, S., \& Richdale, A. (2006). Brief report: Parental descriptions of sleep problems in children with autism, Down syndrome, and Prader-Willi syndrome. Research in Developmental Disabilities, 27(2), 151-161.

Courcy, I., \& des Rivières-Pigeon, C. (2012). $\mathrm{ICl}$ : Des effets positifs pour toutes la famille. L' Express, 38-39. Repéré à http://www.autisme.qc.ca/assets/files/ TSA/methodes_educatives/Effetspositifs-ICl.pdf

Courcy, I., \& des Rivières-Pigeon, C. (2013). Déterminants sociaux de la santé et symptômes dépressifs chez les mères de jeunes enfants ayant un trouble du spectre de l'autisme (TSA). Canadian Journal of Community Mental Health, 32(3), 29-49.

Crockett, J. L., Fleming, R. K., Doepke, K. J., \& Stevens, J. S. (2007). Parent training: Acquisition and generalization of discrete trials teaching skills with parents of children with autism. Research in Developmental Disabilities, 28(1), 23-36.

Davis, N. O., \& Carter, A. S. (2008). Parenting stress in mothers and fathers of toddlers with autism spectrum disorders: Associations with child characteristics. Journal of Autism and Developmental Disorders, 38(7), 1278-1291.

des Rivières-Pigeon, C., \& Courcy, I. (2014). Autisme et TSA: Quelle réalité pour les parents au Québec ? Québec : Presses de l'Université du Québec

Direction générale de la formation continue, \& Université Laval. (2003a). Certificat sur mesure en intervention sur les troubles envahissants du développement Repéré à http://www.dgfc.ulaval. ca/formations/programmes-etudes/ certificat-sur-mesure-intervention-surles-63/corporatif/

Direction générale de la formation continue, \& Université Laval. (2003b). DESS sur mesure en supervision de l'intervention auprès des personnes présentant un trouble envahissant du développement (TED). Repéré à http://www.dgfc.ulaval. $\mathrm{ca} /$ formations/programmes-etudes/ diplome-etudes-superieures-surmesure-supervision-81/corporatif/

Dunn, M. E., Burbine, T., Bowers, C. A., \& Tantleff-Dunn, S. (2001). Moderators of stress in parents of children with autism. Community Mental Health Journal, 37(1), 39-52.

Eikeseth, S. (2011). Intensive Early Intervention. Dans J. L. Matson \& P. Sturmey (dir.), International handbook of autism and pervasive developmental disorders (p. 321-338). New York: Springer. 
Eikeseth, S., Klintwall, L., Jahr, E., \& Karlsson, P. (2012). Outcome for children with autism receiving early and intensive behavioral intervention in mainstream preschool and kindergarten settings. Research in Autism Spectrum Disorders, 6(2), 829-835.

Eisenhower, A., Baker, B. L., \& Blacher, J. (2005). Preschool children with intellectual disability: Syndrome specificity, behaviour problems, and maternal well being. Journal of Intellectual Disability Research, 49(9), 657-671.

Eldevik, S., Hastings, R. P., Hughes, J. C., Jahr, E., Eikeseth, S., \& Cross, S. (2009). Meta-analysis of early intensive behavioral intervention for children with autism. Journal of Clinical Child \& Adolescent Psychology, 38(3), 439450.

Eldevik, S., Hastings, R. P., Hughes, J. C., Jahr, E., Eikeseth, S., \& Cross, S. (2010). Using participant data to extend the evidence base for intensive behavioral intervention for children with autism. American Journal on Intellectual and Developmental Disabilities, 115(5), 381-405.

Eldevik, S., Hastings, R. P., Jahr, E., \& Hughes, J. C. (2012). Outcomes of behavioral intervention for children with autism in mainstream pre-school settings. Journal of Autism and Developmental Disorders, 42(2), 210220.

Estes, A., Munson, J., Dawson, G., Koehler, E., Zhou, X. H., \& Abbott, R. (2009). Parenting stress and psychological functioning among mothers of preschool children with autism and developmental delay. Autism, 13(4), 375.

Fernell, E., Hedvall, Å., Westerlund, J., Höglund Carlsson, L., Eriksson, M., Barnevik Olsson, M., . . . Gillberg, C. (2011). Early intervention in 208 Swedish preschoolers with autism spectrum disorder. A prospective naturalistic study. Research in Developmental Disabilities, 32(6), 2092-2101.

Fombonne, E., Zakarian, R., Bennett, A., Meng, L., \& McLean-Heywood, D.
(2006). Pervasive developmental disorders in Montreal, Quebec, Canada: Prevalence and links with immunizations. Pediatrics, 118(1), 139150.

Forget, J., Schuessler, K., Paquet, A., \& Giroux, N. (2005). Analyse appliquée du comportement et intervention comportementale intensive. Revue québécoise de psychologie, 26(3), 2942.

FQCRDITED. (2003). Plan national de formation en troubles envahissants du développement Repéré le janvier 2014 à http://fqcrdited.org/soutien-alexpertise/formation/1233-2/ - sthash. y6LEgPST.dpuf

Gamache, V., Joly, J., \& Dionne, C. (2011). La fidélité d'implantation du programme québécois d'intervention comportementale intensive destiné aux enfants ayant un trouble envahissant du développement en CRDITED. Revue de psychoéducation, 40(1), 1-23.

Giarelli, E., Souders, M., Pinto-Martin, J., Bloch, J., \& Levy, S. E. (2005). Intervention pilot for parents of children with autistic spectrum disorder. Pediatric Nursing, 31(5), 389-399.

Loi sur les services de santé et les services sociaux, 1991, c. 42, a. 103. C.F.R. (2013).

Granger, S., des Rivières-Pigeon, C., Sabourin, G., \& Forget, J. (2010). Mothers' reports of their involvement in early intensive behavioral intervention. Topics in Early Childhood Special Education, 20(10), 1-10.

Green, C. L., Walker, J. M. T., HooverDempsey, K. V., \& Sandler, H. M. (2007). Parents' motivations for involvement in children's education: An empirical test of a theoretical model of parental involvement. Journal of Educational Psychology, 99(3), 532-544.

Grindle, C. F., \& Remington, B. (2014). Intensive Behavioral Intervention and Family Psychological Adjustment in Autism. Comprehensive Guide to Autism, 97-115. 
Guralnick, M. J. (2000). Early childhood intervention. Focus on Autism and Other Developmental Disabilities, 15(2), 68-79.

Guralnick, M. J. (2001). A developmental systems model for early intervention. Infants and Young Children, 14(2), 1-18.

Guralnick, M. J. (2005). An overview of the developmental systems model for early intervention. Dans M. J. Guralnick (dir.), The Developmental Systems Approach to Early Intervention (p. 3-28). Baltimore: Brookes.

Gurney, J. G., McPheeters, M. L., \& Davis, M. M. (2006). Parental report of health conditions and health care use among children with and without autism: National survey of children's health. Archives of Pediatrics and Adolescent Medicine, 160(8), 825-830.

Hastings, R. P., \& Johnson, E. (2001). Stress in UK families conducting intensive home-based behavioral intervention for their young child with autism. Journal of Autism and Developmental Disorders, 31(3), 327-336.

Hastings, R. P., \& Symes, M. D. (2002). Early intensive behavioral intervention for children with autism: Parental therapeutic self-efficacy. Research in Developmental Disabilities, 23(5), 332341.

Hayes, S. A., \& Watson, S. L. (2013). The impact of parenting stress: A metaanalysis of studies comparing the experience of parenting stress in parents of children with and without autism spectrum disorder. Journal of Autism and Developmental Disorders, 43(3), 629-642.

Hayward, D. W., Gale, C. M., \& Eikeseth, S. (2009). Intensive behavioural intervention for young children with autism: A research-based service model. Research in Autism Spectrum Disorders, 3(3), 571-580.

Johnson, E., \& Hastings, R. P. (2002). Facilitating factors and barriers to the implementation of intensive homebased behavioural intervention for young children with autism. Child: Care, Health and Development, 28(2), 123129.

Jones, T. L., \& Prinz, R. J. (2005). Potential roles of parental self-efficacy in parent and child adjustment: A review. Clinical Psychology Review, 25(3), 341-363.

Karst, J. S., \& Van Hecke, A. V. (2012). Parent and family impact of autism spectrum disorders: A review and proposed model for intervention evaluation. Clinical Child and Family Psychology Review, 15(3), 247-277.

Kuhn, J. C., \& Carter, A. S. (2006). Maternal self-efficacy and associated parenting cognitions among mothers of children with autism. American Journal of Orthopsychiatry, 76(4), 564-575.

Lamontagne, A. J. (2011). Le taux de prévalence des troubles envahissants du développement (TED) Document inédit.

Larsson, E. V. (2008). Intensive early intervention using behavior therapy is the single most widely accepted treatment for autism. Repéré à http:// www.wncbest.org/fileadmin/user_ upload/ieibt-Lovaas.pdf

Larsson, E. V. (2010). ABA and EIBI: Are they evidence-based treatments for autism?

Lazoff, T., Lihong, Z., Piperni, T., \& Fombonne, E. (2010). Prevalence of pervasive developmental disorders among children at the English Montreal School Board. Canadian Journal of Psychiatry, 55(11), 715-720.

Lord, C., \& McGee, J. P. (2001). Educating children with autism. Washington, DC: National Academic Press.

Lovaas, O. I. (1987). Behavioral treatment and normal educational and intellectual functioning in young autistic children. Journal of Consultant and Clinical Psychology, 55(1), 3-9.

Lovaas, O. I., Koegel, R., Simmons, J. Q., \& Long, J. S. (1973). Some generalization and follow-up measures on autistic children in behavior therapy. Journal of Applied Behavior Analysis, 6(1), 131-166. 
Maglione, M. A., Gans, D., Das, L., Timbie, J., \& Kasari, C. (2012). Nonmedical interventions for children with ASD: Recommended guidelines and further research needs. Pediatrics, 130(Supplement 2), S169-S178.

Makrygianni, M. K., \& Reed, P. (2010). A meta-analytic review of the effectiveness of behavioural early intervention programs for children with autistic spectrum disorders. Research in Autism Spectrum Disorders, 4(4), 577-593.

Martins, Y., Young, R. L., \& Robson, D. C. (2008). Feeding and eating behaviors in children with autism and typically developing children. Journal of Autism and Developmental Disorders, 38(10), 1878-1887.

Matson, J. L., \& Nebel-Schwalm, M. S. (2007). Comorbid psychopathology with autism spectrum disorder in children: An overview. Research in Developmental Disabilities, 28(4), 341-352.

Matson, M. L., Mahan, S., \& Matson, J. L. (2009). Parent training: A review of methods for children with autism spectrum disorders. Research in Autism Spectrum Disorders, 3(4), 868-875.

McConachie, H., \& Diggle, T. (2007). Parent implemented early intervention for young children with autism spectrum disorder: A systematic review. Journal of Evaluation in Clinical Practice, 13(1), 120-129.

McGahan, L. (2001). Interventions comportementales chez les enfants d'âge préscolaire atteints d'autisme. Rapport technologique, 18. Repéré à http://www.cadth.ca/media/pdf/105_ autism_tr_f.pdf

McGee, G. G., Morrier, M. J., \& Daly, T. (1999). An incidental teaching approach to early intervention for toddlers with autism. Journal of the Association for Persons with Severe Handicaps.

Mekki, K. (2012). Stress and Coping in Mothers of Children with Autism Spectrum Disorders (Thèse de maîtrise). Université d'Ottawa, Ottawa.
Miles, M. B., \& Huberman, A. M. (2003). Analyse des données qualitatives. Bruxelles : De Boeck Supérieur.

Minjarez, M. B., Williams, S. E., Mercier, E. M., \& Hardan, A. Y. (2011). Pivotal response group treatment program for parents of children with autism. Journal of Autism and Developmental Disorders, 41(1), 92-101.

Montes, G., \& Halterman, J. S. (2007). Psychological functioning and coping among mothers of children with autism: A population-based study. Pediatrics, 119(5), 1040-1046.

National Research Council (2001). Educating children with autism. Washington: The National Academies Press.

New York State Department of Health Early Intervention Program (1999). Clinical Practice Guideline, Autism/Pervasive Developmental Disorders: Assessment and Intervention for Young Children. New York: New York Department of Health.

Newschaffer, C. J., Croen, L. A., Daniels, J., Giarelli, E., Grether, J. K., Levy, S. E., . . . Reaven, J. (2007). The epidemiology of autism spectrum disorders. Annual Review of Public Health, 28, 235-258.

Paillé, P., \& Mucchielli, A. (2008). L'analyse qualitative en sciences humaines et sociales (2e ed.) Paris: Armand Colin.

Perry, A., Cummings, A., Dunn Geier, J., Freeman, N. L., Hughes, S., LaRose, L., .. Williams, J. (2008). Effectiveness of intensive behavioral intervention in a large, community-based program. Research in Autism Spectrum Disorders, 2(4), 621-642.

Peters-Scheffer, N., Didden, R., Korzilius, H., \& Sturmey, P. (2011). A metaanalytic study on the effectiveness of comprehensive ABA-based early intervention programs for children with autism spectrum disorders. Research in Autism Spectrum Disorders, 5(1), 6069. 
Poirier, A. (2008). Processus d'évaluation diagnostique et annonce du diagnostic en trouble envahissant du développement au Québec : expérience et perception des parents.

Poirier, A., \& Goupil, G. (2008). Processus diagnostique des personnes présentant un trouble envahissant du développement au Québec : expérience des parents. Journal on Developmental Disabilities, 14(3), 19-28.

Protecteur du citoyen. (2009). Rapport spécial du protecteur du citoyen. Les services gouvernementaux destinés aux enfants présentant un trouble envahissant du développement: Pour une meilleure continuité dans les services, les approches et les rapports humains. Récupéré de http://www. protecteurducitoyen.qc.ca/fileadmin / medias/pdf/rapports_speciaux/TED.pdf

Protecteur du citoyen. (2012). Rapport spécial du protecteur du citoyen. Les services aux enfants et aux adultes présentant un trouble envahissant du développement : De l'engagement gouvernemental à la réalité. Récupéré de http://www.protecteurducitoyen.qc. ca/fileadmin/medias/pdf/rapports speciaux/2012-05-23_rapport_ted_2. pdf

Reichow, B. (2012). Overview of metaanalyses on early intensive behavioral intervention for young children with autism spectrum disorders. Journal of Autism and Developmental Disorders, 42(4), 512-520.

Rivard, M., Terroux, A., Parent-Boursier, C., \& Mercier, C. (2014). Determinants of stress in parents of children with autism spectrum disorders. Journal of Autism and Developmental Disorders, 1-12.

Sabourin, G., des Rivières-Pigeon, C., \& Granger, S. (2011). La perception des mères quant aux rôles des intervenantes dans les programmes d'intervention comportementale intensive pour les enfants autistes. Revue de psychoéducation, 40(1), 51-70.

Saracino, J., Noseworthy, J., Steiman, M., Reisinger, L., \& Fombonne, E. (2010).
Diagnostic and assessment issues in autism surveillance and prevalence. Journal of Developmental and Physical Disabilities, 22, 317-330.

Sénéchal, C., \& des Rivières-Pigeon, C. (2009). Impact de l'autisme sur la vie des parents. Santé mentale au Québec, 34(1), 245-260.

Siklos, S., \& Kerns, K. A. (2006). Assessing need for social support in parents of children with autism and Down syndrome. Journal of Autism and Developmental Disorders, 36(7), 921933.

Solish, A., \& Perry, A. (2008). Parents' involvement in their children's behavioral intervention programs: Parent and therapist perspectives. Research in Autism Spectrum Disorders, 2(4), 728738.

Twoy, R., Connolly, P., \& Novak, J. (2007). Coping strategies used by parents of children with autism. Journal of the American Academy of Nurse Practitioners, 19(5), 251-260.

Tzanakaki, P., Grindle, C., Hastings, R. P., Carl Hughes, J., Kovshoff, H., \& Remington, B. (2012). How and Why do Parents Choose Early Intensive Behavioral Intervention for their Young Child with Autism ? Education and training in autism and developmental disabilities, 47(1), 58.

Veyssière, A., Bernadoy, C., Dagot, L., \& Goussé, V. (2010). Étude qualitative de l'évolution des stratégies de faire face chez les parents d'adolescents atteints de troubles autistiques. Pratiques Psychologiques, 16(3), 239-248.

Virués-Ortega, J. (2010). Applied behavior analytic intervention for autism in early childhood: Meta-analysis, metaregression and dose-response metaanalysis of multiple outcomes. Clinical Psychology Review, 30(4), 387-399.

Warren, Z., McPheeters, M. L., Sathe, N., Foss-Feig, J. H., Glasser, A., \& VeenstraVanderWeele, J. (2011). A systematic review of early intensive intervention for autism spectrum disorders. Pediatrics, 127(5), e1303-e1311. 
Weintraub, K. (2011). Autism counts. Nature(479), 22-24.

White, N., \& Hastings, R. P. (2004). Social and professional support for parents of adolescents with severe intellectual disabilities. Journal of Applied Research in Intellectual Disabilities, 17(3), 181190.

Wintgens, A., \& Hayez, J. Y. (2006). Guidance psychopédagogique des parents d'enfants atteints d'autisme. La psychiatrie de l'enfant, 49(1), 207-226. 